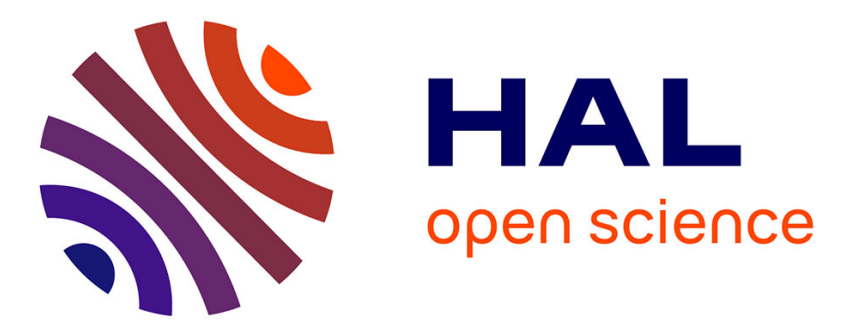

\title{
Consistent tangent operator for an exact Kirchhoff rod model
}

\author{
L. Greco, M. Cuomo
}

\section{To cite this version:}

L. Greco, M. Cuomo. Consistent tangent operator for an exact Kirchhoff rod model. Continuum Mechanics and Thermodynamics, 2014, 17 p. hal-01009187

\section{HAL Id: hal-01009187 \\ https://hal.science/hal-01009187}

Submitted on 17 Jun 2014

HAL is a multi-disciplinary open access archive for the deposit and dissemination of scientific research documents, whether they are published or not. The documents may come from teaching and research institutions in France or abroad, or from public or private research centers.
L'archive ouverte pluridisciplinaire HAL, est destinée au dépôt et à la diffusion de documents scientifiques de niveau recherche, publiés ou non, émanant des établissements d'enseignement et de recherche français ou étrangers, des laboratoires publics ou privés. 
L. Greco - M. Cuomo

\title{
Consistent tangent operator for an exact Kirchhoff rod model
}

\begin{abstract}
In the paper, it is considered an exact spatial Kirchhoff rod structural model. The configuration space for this model that has dimension 4 is obtained considering an ad hoc split of the rotation operator that implicitly enforces the constraints on the directors. The tangent stiffness operator, essential for the nonlinear numerical simulations, has been studied. It has been obtained as second covariant gradient of the internal energy functional for the considered structural model that preserves symmetry for any configuration, either equilibrated or not. The result has been reached evaluating the Levi-Civita connection for the tangent space of the configuration manifold. The results obtained extend to the case of Kirchhoff-Love rods those presented by Simo (Comput Methods Appl Mech Eng 49:55-70, 1985) for Timoshenko rods. Given the different structure of the tangent spaces in this case, it has been necessary to introduce a specific metric that accounts for the rotation of the intrinsic triad due to the change of the position of the centroid axis of the rod.
\end{abstract}

Keywords Nonlinear Kirchhoff-Love rods · Tangent stiffness · Symmetry of geometric stiffness · Levi-Civita connection

\section{Introduction}

Nonlinear beam models date back to Euler's elastica, a planar beam experiencing only bending deformations. Kirchhoff rod extends the model to spatial beams with torsional deformation. According to Love, much of the developments in the theory of elastic rods is based on the discovery made by Kirchhoff that the equations for the equilibrium configurations of an elastic rod that is straight and prismatic when it is unstressed are the same as the Lagrange equations for the spinning top. Love extended the theorem to naturally curved rods and added axial strain to the model, while the cross section remains orthogonal to the beam axis. An extensive bibliographic tradition that goes back to Reissner, Antman, and Simo [2, 8,37,51,53] refers to Kirchhoff model of rods as a space curve equipped with an adaptive frame, including bending, torsion, and axial straining, but not shear strains. Additional deformation fields have also been introduced for accounting for the deformation of the cross section.

In his celebrated and fundamental article of 1985 , Simo introduced the term geometrically exact finite strain beam theory referring to a model using sectional strain measures that are exact regardless of the magnitude of displacements, rotations, and strains. He considered a shear deformable beam, as most of the numerical models developed since then have done. Geometrically exact beam theories require that arbitrarily large 3D finite rotations be accurately accounted for, both in the continuum model and in the computational discretization. Many numerical models have proposed different discretization schemes for the rotations [10,25,35], and

Communicated by Francesco dell' Isola and Giuseppe Piccardo.

L. Greco · M. Cuomo (四)

University of Catania, Catania, Italy

E-mail: mcuomo@ dica.unict.it 
more recently discrete differential geometry has also been proposed as a tool for developing consistent exact numerical models of rods [8].

Beam theories can be obtained employing a reduction process from the $3 \mathrm{D}$ continuum, or introducing specific assumptions for the kinematics, and for the strain energy, the latter models usually are referred to as structural models. In the paper, we will follow the latter strategy. The definition of the structural model implies therefore the specification of its configuration space, the set of the possible geometrical configurations $\mathcal{V}$ of the model endowed with a smooth chart (generally, an atlas). Smoothness indicates that the change of coordinates between any two charts defined on the set $\mathcal{V}$ is differentiable. The dimension of the configuration space characterizes the structural model at hand. The space of the admissible variations of the configuration, necessary for setting up the principle of virtual work, is given by the tangent bundle to $\mathcal{V}$. This aspect is fundamental in the nonlinear analysis of space rods, since the tangent operator is needed in the implementation of the iterative method of solution of the nonlinear equilibrium equations.

In a geometrically exact beam model, the configuration of the space rod is mapped by the position of the centerline and by the finite rotations of three directors attached to the beam axis. In this way, spatial rod models represent a one-dimensional particularization of the general polar continuum, also known as Cosserat continuum. In the case when the rotations of the directors are independent, we get the Timoshenko beam or its generalization to the spatial case ([53-56]). Analogously, a three-dimensional nonlinear polar model with drilling rotations was developed for shells in [22-24,28,33,34,47], and without drilling rotation in $[3,9,60,61]$. The space $S O$ (3) of finite rotational degrees of freedom is, however, noncommutative, so that their increments cannot be simply added, for details see $[6,7,52,57]$. This problem has been strongly addressed in computational mechanics by Crisfield and Jelenic [12] and by Jelenic and Crisfield [36] who, in order to overcome the problem, proposed to interpolate the local rotations with respect to a corotational frame. They also pointed out that the strain measures must remain unaffected by a superimposed rigid rotation of the configuration (objectivity) also in the interpolation scheme. Later alternative methods to the corotational scheme have been proposed (see [25,32]). A representation based on the natural (Bishop) frame for shear undeformable space rods under Kirchhoff-Love hypotheses has been introduced by Langer and Singer [37] and subsequently used by Bergou et al. as starting point for an exact rod model built up on discrete differential geometry [8].

As a consequence of the fact that the configuration space for the rod model is a non-Euclidean manifold, the structural coefficients of the space (i.e., the Christoffel symbols of the connection) do not vanish; therefore, the directional Gateaux derivative loses the covariant character. The Gateaux derivative is usually employed in continuum mechanics for obtaining the variation of the configuration, while the second Gateaux derivative of the total potential energy yields the tangent stiffness operator needed in incremental analyses. Since the latter does not represent the second covariant gradient, the tangent operator calculated in this way loses its symmetry properties.

The issue was raised by Simo [58,59], who first noted that the tangent operator evaluated using the Gateaux derivative is symmetric only at an equilibrium configuration, if the load is conservative. He also described a procedure for evaluating the Hessian of the total potential energy that does not require the explicit knowledge of the symbols of the connection that amounts to a straightforward symmetrization of the operator obtained performing the directional derivative. These facts are indicated in detail in [13]. Later, Jelenic [35] and others authors afterward used this conclusion. The evaluation of the symmetric tangent operator for an exact structural model is a necessary requirement in order to extend the formulation to beams composed by materials with local symmetries, as occurs in functionally graded beams (see for instance [21] for the use of material local symmetry groups in structural models, and [11] for the theory of functionally graded beams).

In a general sense, in order to obtain the consistent tangent operator of the energy functional, it is needed to define a connection on the tangent bundle to the configuration manifold and then to calculate the second covariant gradient. It is symmetric only if the connection is torsion-free; therefore, the unique torsion-free connection consistent with the adopted metric has to be chosen (i.e., the Levi-Civita connection). This fact, consisting in the fundamental theorem of the Riemman's geometry, already present in $[13,58,59]$, allows to overcome the noncommutative nature of the rotation group.

The cited works refer to shear deformable rods, whose numerical implementation in a finite element (FE) scheme presents, as it is well-known, shear locking. Kirchhoff-Love rod models are able to avoid shear locking, so they can be effectively used in modeling bending and torsion-dominated nonlinear problem. However, membrane and torsional locking may be present, as it has been illustrated in [15] where a family of mixed finite elements for curved elements, based on isogeometric interpolation, has been introduced that avoids both membrane locking and the effect of flexure locking on the twisting energy terms. Recently, it has been shown in [26] that using a hierarchic shell approach, it is possible to develop a numerical model free of shear locking, 
(obtaining a sort of switch element) affected only by membrane locking. On this line, the unshearable model used in this work can be considered an interesting base for developing efficient numerical models for rods. Kirchhoff-Love model offers several other advantages, since there is no need of assuming any constitutive behavior for the shear that would introduce significant errors in the theory. Among the many new interesting problems of mechanical engineering that can be effectively modeled in this way, we recall the simulation of living tissues, like cilia, of wind-induced loads on eolic turbines, the analysis of micro piezoelectric actuators, etc. [1,4,14,16,17, 19,29,45,49,50,62].

The configuration manifold of a Kirchhoff-Love model is different from the one used in the Timoshenko model, since the bending rotations are not independent and are obtained from the position field. Therefore, the configuration space that in the former case is $\mathbb{R}^{3} \times \mathrm{SO}(3)$ becomes $\mathbb{R}^{3} \times \mathrm{S}^{1}$, so that the derivation of the Hessian outlined in Simo's paper cannot be directly applied and needs some adjustments.

In this work, we analyze in detail and obtain the tangent stiffness operator for the exact spatial KirchhoffLove rod model presented in $[30,31]$ that is characterized by intrinsically enforcing the Kirchhoff-Love constraints through a split of the rotation operator that defines the unit triad attached to the centroid curve. Differently from standard approaches that adopts the Frenet triad, we consider a more general intrinsic triad that in general is not torsion-free. In particular, we introduce a first rotation operator, indicated with $\boldsymbol{\Lambda} \in \mathrm{S}^{2}$, defined as a rotation from the reference to the current unit tangents (i.e., this first unit operator is a finite rotation without drilling around the reference centroid axis). By means of the $\boldsymbol{\Lambda}$ operator, it is defined a service unit triad that successively can be rotated, through the operator $\boldsymbol{R} \in \mathrm{S}^{1}$, around the current unit tangent vector in order to define the current unit triad. The rotation operator $\boldsymbol{\Lambda}$ is fully characterized by the displacement field. In this way, the strain energy of the rod, in particular the bending energy term, depends on the second covariant derivative of the centroid displacement, differently from what happens in shear deformable beams. The required generalization is obtained on the basis of arguments generalizing or paralleling those presented for instance in $[5,18,20,27]$. The tangent operator obtained in this way is fundamental for the iterative incremental analysis; further, higher expansion of the potential is necessary for the study of the post-critical behavior, by means of perturbation methods, as shown in the many contributes given by Luongo and coworkers, [38-42]. The method described in the paper can be usefully employed for these higher expansions.

\section{Kinematics}

In this section, a brief summary of the finite kinematics of a Kirchhoff-Love rod and of its tangent form is given, which is described in detail in [30,31]. Following this approach, the rod is defined by its centroid curve and by the directors lying in the plane orthogonal to the centroid line.

\subsection{Reference configuration}

The centroid line of the rod is mapped from an interval $\mathcal{A}$ by means of the position vector $\boldsymbol{p}_{0}: \mathcal{A} \subset \mathbb{R} \rightarrow \mathbb{R}^{3}$. The tangent to the reference position is

$$
\hat{\boldsymbol{t}}_{0}:=\frac{\mathrm{d} \boldsymbol{p}_{0}}{\mathrm{~d} S}, \quad\left\|\hat{\boldsymbol{t}}_{0}\right\|=1,
$$

where $S$ is the arc length of the reference centroid curve. Let $\hat{\boldsymbol{n}}_{0}: \mathcal{A} \rightarrow \mathbb{R}^{3}$ be a director orthogonal to $\hat{\boldsymbol{t}}_{0}$, and $\hat{\boldsymbol{v}}_{0}:=\hat{\boldsymbol{t}}_{0} \times \hat{\boldsymbol{n}}_{0}$, so that the intrinsic unit triad of the rod in the reference configuration is given by $\left\{\hat{\boldsymbol{t}}_{0}, \hat{\boldsymbol{n}}_{0}, \hat{\boldsymbol{v}}_{0}\right\}$. The normal vector field $\hat{\boldsymbol{n}}_{0}(S)$ is chosen arbitrarily. In particular, the natural frame, defined as a torsion-free frame, can be selected, or any other convenient choice is possible. In the paper, we refer to a generic reference frame, with an initial twist.

The curvatures of the centerline are defined on the reference configuration as

$$
\chi_{n 0}=-\frac{\mathrm{d} \hat{\boldsymbol{t}}_{0}}{\mathrm{~d} S} \cdot \hat{\boldsymbol{v}}_{0}, \quad \chi_{\nu 0}=\frac{\mathrm{d} \hat{\boldsymbol{t}}_{0}}{\mathrm{~d} S} \cdot \hat{\boldsymbol{n}}_{0}, \quad \chi_{t 0}=\frac{\mathrm{d} \hat{\boldsymbol{n}}_{0}}{\mathrm{~d} S} \cdot \hat{\boldsymbol{v}}_{0}
$$

The position of the generic point of the rod is given by

$$
{\stackrel{*}{\boldsymbol{p}_{0}}}=\boldsymbol{p}_{0}+\vartheta^{n} \hat{\boldsymbol{n}}_{0}+\vartheta^{v} \hat{\boldsymbol{v}}_{0},
$$

where $\vartheta^{n}$ and $\vartheta^{v}$ are the quota functions, independent of the arc length. 


\subsection{Current configuration}

The position vector for the points of the centerline is $\boldsymbol{p}: \mathcal{A} \rightarrow \mathbb{R}^{3}$. Denoting by $\boldsymbol{u}: \mathcal{A} \rightarrow \mathbb{R}^{3}$ the displacement field of the centroid curve, the position vector is given by

$$
p:=p_{0}+u
$$

and the tangent vector is

$$
t:=\frac{\mathrm{d} \boldsymbol{p}}{\mathrm{d} S}, \quad \hat{\boldsymbol{t}}:=\frac{\boldsymbol{t}}{\|\boldsymbol{t}\|}, \quad\|\boldsymbol{t}\|:=\sqrt{\boldsymbol{t} \cdot \boldsymbol{t}}
$$

The orientation of the current triad is obtained applying two subsequent rotations. The first rotation $\boldsymbol{\Lambda}\left(\hat{\boldsymbol{t}}_{0}, \hat{\boldsymbol{t}}\right)(S)$ brings the unit tangent vector $\left\{\hat{\boldsymbol{t}}_{0}(S)\right.$ on $\left.\hat{\boldsymbol{t}}(S)\right\}$; that is, it is a rotation without drilling around the tangent to the reference centroid line. The axial vector of this rotation is $\hat{\boldsymbol{t}}_{0} \times \hat{\boldsymbol{t}}$, and Rodrigues formula gives the representation:

$$
\boldsymbol{\Lambda}\left(\hat{\boldsymbol{t}}_{0}, \hat{\boldsymbol{t}}\right):=\left(\hat{\boldsymbol{t}}_{0} \cdot \hat{\boldsymbol{t}}\right) \boldsymbol{I}+\left(\hat{\boldsymbol{t}}_{0} \times \hat{\boldsymbol{t}}\right) \times \boldsymbol{I}+\frac{\left(\hat{\boldsymbol{t}}_{0} \times \hat{\boldsymbol{t}}\right) \otimes\left(\hat{\boldsymbol{t}}_{0} \times \hat{\boldsymbol{t}}\right)}{1+\hat{\boldsymbol{t}}_{0} \cdot \hat{\boldsymbol{t}}}
$$

The rotation operator, $\boldsymbol{\Lambda}$, defines an intermediate unit triad on the orthogonal plane to the current centroid curve as

$$
\left\{\hat{\boldsymbol{t}}, \hat{\boldsymbol{n}}^{\mathrm{b}}, \hat{\boldsymbol{v}}^{\mathrm{b}}\right\}:=\left\{\boldsymbol{\Lambda} \hat{\boldsymbol{t}}_{0}, \boldsymbol{\Lambda} \hat{\boldsymbol{n}}_{0}, \boldsymbol{\Lambda} \hat{\boldsymbol{v}}_{0}\right\}
$$

An additional rotation operator, $\boldsymbol{R}(\hat{\boldsymbol{t}}, \phi)(S)$, is then applied, given by a rotation $\phi(S)$ of the intermediate triad around the current tangent to the rod,

$$
\boldsymbol{R}(\hat{\boldsymbol{t}}, \phi):=\boldsymbol{I}+\sin [\phi](\hat{\boldsymbol{t}} \times \boldsymbol{I})+(1-\cos [\phi])(\hat{\boldsymbol{t}} \times(\hat{\boldsymbol{t}} \times \boldsymbol{I})) .
$$

Notice that in this way, the Kirchhoff-Love constraints are automatically enforced. The spatial triad for the Kirchhoff rod is then

$$
\{\hat{\boldsymbol{t}}, \hat{\boldsymbol{n}}, \hat{\boldsymbol{v}}\}:=\left\{\hat{\boldsymbol{t}}, \boldsymbol{R} \hat{\boldsymbol{n}}^{\mathrm{b}}, \boldsymbol{R} \hat{\boldsymbol{v}}^{\mathrm{b}}\right\}=\left\{\boldsymbol{\Lambda} \hat{\boldsymbol{t}}_{0}, \boldsymbol{R} \boldsymbol{\Lambda} \hat{\boldsymbol{n}}_{0}, \boldsymbol{R} \boldsymbol{\Lambda} \hat{\boldsymbol{v}}_{0}\right\}
$$

The curvatures of the spatial configuration are defined in a similar way to Eq. (2):

$$
\begin{aligned}
\chi_{t} & =\frac{\mathrm{d} \hat{\boldsymbol{n}}}{\mathrm{d} S} \cdot \hat{\boldsymbol{v}}, \quad \chi_{n}=-\frac{\mathrm{d} \boldsymbol{t}}{\mathrm{d} S} \cdot \hat{\boldsymbol{v}}, \quad \chi_{v}=\frac{\mathrm{d} \boldsymbol{t}}{\mathrm{d} S} \cdot \hat{\boldsymbol{n}} \\
\boldsymbol{b} & =\hat{\boldsymbol{t}} \times \frac{\mathrm{d} \boldsymbol{t}}{\mathrm{d} S}=\chi_{n} \hat{\boldsymbol{n}}+\chi_{v} \hat{\boldsymbol{v}} .
\end{aligned}
$$

The spatial position of a generic point of the rod is obtained as

$$
\stackrel{*}{p}=\boldsymbol{p}+\vartheta^{n} \hat{\boldsymbol{n}}+\vartheta^{v} \hat{\boldsymbol{v}}
$$

The configuration space of the Kirchhoff rod is then given by

$$
\mathbb{M}:=\left\{\boldsymbol{\varphi}=(\boldsymbol{p}, \boldsymbol{\Lambda}, \boldsymbol{R}):[0, L] \rightarrow\left(\mathbb{R}^{3} \times \mathrm{S}^{2}\right) \times \mathrm{S}^{1}\right\} .
$$

Notice that the dimension of the manifold $\mathbb{M}$ is 4 , since the position $\boldsymbol{p}$ and the rotation $\boldsymbol{\Lambda}$ can both be expressed as functions of the displacement field $\boldsymbol{u}$. 


\subsection{Tangent kinematics}

In this section, we derive the variation field for the configuration of the rod. If $\boldsymbol{p}_{\epsilon}$ is a 1-parameter admissible family of positions of the centerline, such that $\boldsymbol{p}_{\epsilon=0}=\boldsymbol{p}$, the variation of the centerline position $\tilde{\boldsymbol{u}}: \mathbb{M} \rightarrow \mathrm{T}_{\boldsymbol{p}} \mathbb{M}$ is defined as

$$
\tilde{\boldsymbol{u}}:=\left.\frac{\mathrm{d} \boldsymbol{p}_{\epsilon}}{\mathrm{d} \epsilon}\right|_{\epsilon=0} .
$$

The variations of the rotations are defined in a similar way. Let $\boldsymbol{Q}_{\epsilon}=\boldsymbol{R}_{\epsilon} \boldsymbol{\Lambda}_{\epsilon}$ be a curve of admissible rotations for the rod, with $\boldsymbol{\Lambda}_{\epsilon}=\boldsymbol{\Lambda}\left(\hat{\boldsymbol{t}}_{0}, \hat{\boldsymbol{t}}_{\epsilon}\right)$ and $\boldsymbol{R}_{\epsilon}=\boldsymbol{R}\left(\phi_{\epsilon}, \hat{\boldsymbol{t}}_{\epsilon}\right)$, then the variations are given by

$$
\tilde{\boldsymbol{Q}}:=\left.\frac{\mathrm{d} \boldsymbol{Q}_{\epsilon}}{\mathrm{d} \epsilon}\right|_{\epsilon=0}=\tilde{\boldsymbol{R}} \boldsymbol{\Lambda}+\boldsymbol{R} \tilde{\boldsymbol{\Lambda}}, \quad \tilde{\boldsymbol{R}}:=\left.\frac{\mathrm{d} \boldsymbol{R}_{\epsilon}}{\mathrm{d} \epsilon}\right|_{\epsilon=0}, \quad \tilde{\boldsymbol{\Lambda}}:=\left.\frac{\mathrm{d} \boldsymbol{\Lambda}_{\epsilon}}{\mathrm{d} \epsilon}\right|_{\epsilon=0} .
$$

whose spatial representation is given by (see [31] for details)

$$
\begin{aligned}
\tilde{\boldsymbol{Q}} \boldsymbol{Q}^{T} & =\boldsymbol{R} \tilde{\boldsymbol{\Lambda}} \boldsymbol{\Lambda}^{T} \boldsymbol{R}^{T}+\tilde{\boldsymbol{R}} \boldsymbol{R}^{T}, \\
& =(\tilde{\hat{\boldsymbol{t}}} \otimes \hat{\boldsymbol{t}}-\hat{\boldsymbol{t}} \otimes \tilde{\hat{t}})+\tilde{\phi} \hat{\boldsymbol{t}} \times \boldsymbol{I},
\end{aligned}
$$

with

$$
\tilde{\phi}:=\left.\frac{\mathrm{d} \phi_{\epsilon}}{\mathrm{d} \epsilon}\right|_{\epsilon=0}, \quad \tilde{\hat{\boldsymbol{t}}}:=\left.\frac{\mathrm{d} \hat{\boldsymbol{t}}_{\epsilon}}{\mathrm{d} \epsilon}\right|_{\epsilon=0} .
$$

The axial vector of the rotation (15) is the spin:

$$
\tilde{\boldsymbol{\omega}}=\tilde{\phi} \hat{\boldsymbol{t}}+\tilde{\boldsymbol{\omega}}_{b}, \quad \tilde{\boldsymbol{\omega}}_{b}=\tilde{\omega}_{n} \hat{\boldsymbol{n}}+\tilde{\omega}_{\nu} \hat{\boldsymbol{v}} .
$$

The bending rotation $\tilde{\boldsymbol{\omega}}_{b}$, accounting for the Kirchhoff constraints, is given by the following:

$$
\tilde{\omega}_{n}=-\frac{1}{\|\boldsymbol{t}\|} \frac{\mathrm{d} \tilde{\boldsymbol{u}}}{\mathrm{d} S} \cdot \hat{\boldsymbol{v}}, \quad \tilde{\omega}_{\nu}=\frac{1}{\|\boldsymbol{t}\|} \frac{\mathrm{d} \tilde{\boldsymbol{u}}}{\mathrm{d} S} \cdot \hat{\boldsymbol{n}} .
$$

The tangent space to a configuration $\varphi$ is then

$$
\mathrm{T}_{\varphi} \mathbb{M}:=\left\{\tilde{\boldsymbol{\varphi}}:=\left(\tilde{\boldsymbol{u}}, \tilde{\boldsymbol{\omega}}_{b}, \tilde{\phi}\right):[0, L] \rightarrow\left(\mathbb{R}^{3} \times \mathrm{SO}(2)\right) \times \mathrm{SO}(1)\right\} .
$$

\section{Equilibrium operator}

Assuming a linear elastic constitutive behavior (as it is done in [30,54]) and approximating the transport operator for the stresses along the normal directors with the identity, the elastic potential energy of the rod takes the form

$$
\psi_{\mathrm{int}}(\boldsymbol{\varphi})=\frac{1}{2} \int_{0}^{L}\left(E A \varepsilon^{2}(\boldsymbol{\varphi})+E I_{n} \Delta \chi_{n}^{2}(\varphi)+E I_{\nu} \Delta \chi_{v}^{2}(\varphi)+G J_{t} \Delta \chi_{t}^{2}(\varphi)\right) \mathrm{d} S,
$$

where the finite strain measures are respectively

$$
\begin{gathered}
\varepsilon(\varphi):=\frac{\boldsymbol{t} \cdot \boldsymbol{t}-1}{2}, \quad \Delta \chi_{t}(\boldsymbol{\varphi}):=\frac{\mathrm{d} \hat{\boldsymbol{n}}}{\mathrm{d} S} \cdot \hat{\boldsymbol{v}}-\frac{\mathrm{d} \hat{\boldsymbol{n}}_{0}}{\mathrm{~d} S} \cdot \hat{\boldsymbol{v}}_{\mathbf{0}} \\
\Delta \chi_{n}(\boldsymbol{\varphi}):=\frac{\mathrm{d} \hat{\boldsymbol{v}}}{\mathrm{d} S} \cdot \boldsymbol{t}-\frac{\mathrm{d} \hat{\boldsymbol{v}}_{0}}{\mathrm{~d} S} \cdot \hat{\boldsymbol{t}}_{0}, \quad \Delta \chi_{v}(\boldsymbol{\varphi}):=-\left(\frac{\mathrm{d} \hat{\boldsymbol{n}}}{\mathrm{d} S} \cdot \boldsymbol{t}-\frac{\mathrm{d} \hat{\boldsymbol{n}}_{0}}{\mathrm{~d} S} \cdot \hat{\boldsymbol{t}}_{0}\right) .
\end{gathered}
$$

A conservative potential $\psi_{\text {ext }}$ is assumed for the external loads. 


\subsection{Weak formulation}

The first Gateaux derivative of the total potential energy $\psi_{\text {int }}+\psi_{\text {ext }}$ gives the weak formulation of the equilibrium equations. In the following, in order to simplify the notation, the symbol indicating the directional Gateaux derivative is substituted with

$$
\partial(\bullet)=\left.\frac{\mathrm{d}(\bullet)}{\mathrm{d} \epsilon}\right|_{\epsilon=0}
$$

The internal virtual power is then

$$
\partial_{\varphi} \psi_{\mathrm{int}}(\varphi)=\int_{0}^{L}\left(N(\varphi) \tilde{\varepsilon}(\varphi)+M_{n}(\varphi) \tilde{\chi}_{n}(\varphi)+M_{\nu}(\varphi) \tilde{\chi}_{\nu}(\varphi)+M_{t}(\varphi) \tilde{\chi}_{t}(\varphi)\right) \mathrm{d} S
$$

where the constitutive relations and the variations of the strain measures are

$$
\begin{aligned}
& N(\boldsymbol{\varphi})=E A \varepsilon(\varphi), \quad \tilde{\varepsilon}(\boldsymbol{\varphi}):=\left.\frac{\mathrm{d} \varepsilon\left(\boldsymbol{\varphi}_{\epsilon}\right)}{\mathrm{d} \epsilon}\right|_{\epsilon=0}=\frac{\mathrm{d} \tilde{\boldsymbol{u}}}{\mathrm{d} S} \cdot \boldsymbol{t} \\
& M_{n}(\boldsymbol{\varphi})=E I_{n} \Delta \chi_{n}(\boldsymbol{\varphi}), \quad \tilde{\chi}_{n}(\boldsymbol{\varphi}):=\left.\frac{\mathrm{d} \chi_{n}\left(\boldsymbol{\varphi}_{\epsilon}\right)}{\mathrm{d} \epsilon}\right|_{\epsilon=0}=\left(-\frac{d^{2} \tilde{\boldsymbol{u}}}{\mathrm{d} S^{2}}+\tilde{\phi} \boldsymbol{b}\right) \cdot \hat{\boldsymbol{v}} \\
& M_{v}(\boldsymbol{\varphi})=E I_{v} \Delta \chi_{v}(\boldsymbol{\varphi}), \quad \tilde{\chi}_{v}(\boldsymbol{\varphi}):=\left.\frac{\mathrm{d} \chi_{v}\left(\boldsymbol{\varphi}_{\epsilon}\right)}{\mathrm{d} \epsilon}\right|_{\epsilon=0}=\left(\frac{d^{2} \tilde{\boldsymbol{u}}}{\mathrm{d} S^{2}}-\tilde{\phi} \boldsymbol{b}\right) \cdot \hat{\boldsymbol{n}} \\
& M_{t}(\boldsymbol{\varphi})=G J_{t} \Delta \chi_{t}(\boldsymbol{\varphi}), \quad \tilde{\chi}_{t}(\boldsymbol{\varphi}):=\left.\frac{\mathrm{d} \chi_{t}\left(\boldsymbol{\varphi}_{\epsilon}\right)}{\mathrm{d} \epsilon}\right|_{\epsilon=0}=\frac{\mathrm{d} \tilde{\phi}}{\mathrm{d} S}+\frac{1}{\|\boldsymbol{t}\|^{2}} \boldsymbol{b} \cdot \frac{\mathrm{d} \tilde{\boldsymbol{u}}}{\mathrm{d} S}
\end{aligned}
$$

in which the second covariant line gradient is defined as

$$
\begin{aligned}
\frac{d^{2} \tilde{\boldsymbol{u}}}{\mathrm{d} S^{2}} & =\|\boldsymbol{t}\| \frac{d}{\mathrm{~d} S}\left(\frac{1}{\|\boldsymbol{t}\|} \frac{\mathrm{d} \tilde{\boldsymbol{u}}}{\mathrm{d} S}\right) \\
& =\frac{d}{\mathrm{~d} S}\left(\frac{\mathrm{d} \tilde{\boldsymbol{u}}}{\mathrm{d} S}\right)-\left(\frac{1}{\|\boldsymbol{t}\|} \frac{\mathrm{d}\|\boldsymbol{t}\|}{\mathrm{d} S}\right) \frac{\mathrm{d} \tilde{\boldsymbol{u}}}{\mathrm{d} S}
\end{aligned}
$$

The variation of the strain measures can be cast in the equivalent form:

$$
\left(\begin{array}{c}
\tilde{\varepsilon} \\
\hat{\boldsymbol{t}} \times \tilde{\chi} \\
\tilde{\chi}_{t}
\end{array}\right)=\left(\begin{array}{cc}
\boldsymbol{t} \cdot \frac{d}{\mathrm{~d} S} & 0 \\
-(\boldsymbol{I}-\hat{\boldsymbol{t}} \otimes \hat{\boldsymbol{t}}) \frac{d^{2}}{\mathrm{~d} S^{2}} & \boldsymbol{b} \\
\frac{\boldsymbol{b}}{\|\boldsymbol{t}\|^{2}} \cdot \frac{d}{\mathrm{~d} S} & \frac{d}{\mathrm{~d} S}
\end{array}\right)\left(\begin{array}{l}
\tilde{\boldsymbol{u}} \\
\tilde{\phi}
\end{array}\right)
$$

Notice that the Gateaux derivative that we have performed coincides with the covariant derivative, since $\psi_{\text {int }}$ is a scalar.

\subsection{Strong formulation}

Applying the Green-Gauss-Ostrogradsky's formula to the internal power, we obtain the first directional derivative of the strain energy potential $\psi$ along the generic tangent direction $\tilde{\varphi} \in \mathrm{T}_{\varphi} \mathbb{M}$, whose stationarity conditions yield the equilibrium equations of the rod:

$$
\left\{\partial_{\tilde{\boldsymbol{u}}} \psi, \partial_{\tilde{\phi}} \psi\right\}\left(\begin{array}{c}
\tilde{\boldsymbol{u}} \\
\tilde{\phi}
\end{array}\right)=\{0,0\}, \quad \forall \tilde{\boldsymbol{u}}, \forall \tilde{\phi}
$$


The strong form of the equations is as follows:

$$
\begin{aligned}
& \partial_{\tilde{\boldsymbol{u}}}(\psi)=\int_{0}^{L}\left\{-\frac{d}{\mathrm{~d} S}\left[N \boldsymbol{t}+\frac{1}{\|\boldsymbol{t}\|}\left(\frac{d}{\mathrm{~d} S}(\|\boldsymbol{t}\| \hat{\boldsymbol{t}} \times \boldsymbol{M})+M_{t} \frac{\boldsymbol{b}}{\|\boldsymbol{t}\|}\right)\right]-\boldsymbol{f}\right\} \mathrm{d} S \\
& \partial_{\tilde{\phi}}(\psi)=\int_{0}^{L}\left\{-\frac{d M_{t}}{\mathrm{~d} S}+\hat{\boldsymbol{t}} \times \boldsymbol{M} \cdot \boldsymbol{b}-m_{t}\right\} \mathrm{d} S .
\end{aligned}
$$

with the boundary conditions

$$
\begin{aligned}
& N_{\text {eff }}(L)=f_{t}(L), \quad \text { or } \quad \dot{u}_{t}(L)=0 \text {, } \\
& -N_{\text {eff }}(0)=f_{t}(0), \quad \text { or } \quad \dot{u}_{t}(0)=0 \text {, } \\
& T_{n}(L)=f_{n}(L), \quad \text { or } \quad \dot{u}_{n}(L)=0, \\
& -T_{n}(0)=f_{n}(0), \quad \text { or } \quad \dot{u}_{n}(0)=0 \text {, } \\
& T_{v}(L)=f_{v}(L), \quad \text { or } \quad \dot{u}_{v}(L)=0 \text {, } \\
& -T_{v}(0)=f_{v}(0), \quad \text { or } \quad \dot{u}_{v}(0)=0,
\end{aligned}
$$

where $f_{t}=\hat{\boldsymbol{t}} \cdot \boldsymbol{f}, f_{n}=\hat{\boldsymbol{n}} \cdot \boldsymbol{f}$, and $f_{v}=\hat{\boldsymbol{v}} \cdot \boldsymbol{f}$, the effective axial stress resultant for the rod $N_{\text {eff }}$ and the two shear components are given by

$$
\begin{aligned}
N_{\text {eff }} & :=\|\boldsymbol{t}\| N+M_{n} \frac{\chi_{n}}{\|\boldsymbol{t}\|}+M_{v} \frac{\chi_{v}}{\|\boldsymbol{t}\|}, \\
-T_{n} & :=\frac{1}{\|\boldsymbol{t}\|} \frac{d}{\mathrm{~d} S}\left(\|\boldsymbol{t}\| M_{v}\right)+M_{n} \chi_{t}-\frac{M_{t}}{\|\boldsymbol{t}\|^{2}} \chi_{n}, \\
T_{v} & :=\frac{1}{\|\boldsymbol{t}\|} \frac{d}{\mathrm{~d} S}\left(\|\boldsymbol{t}\| M_{n}\right)-M_{\nu} \chi_{t}+\frac{M_{t}}{\|\boldsymbol{t}\|^{2}} \chi_{v},
\end{aligned}
$$

and

$$
\begin{gathered}
M_{t}(L)=m_{t}(L), \quad \text { or } \quad \dot{\phi}(L)=0, \\
-M_{t}(0)=m_{t}(0), \quad \text { or } \dot{\phi}(0)=0, \\
\|\boldsymbol{t}\| M_{n}(L)=m_{n}(L), \quad \text { or } \quad \dot{\omega}_{n}(L)=0, \\
-\|\boldsymbol{t}\| M_{n}(0)=m_{n}(0), \quad \text { or } \quad \dot{\omega}_{n}(0)=0, \\
\|\boldsymbol{t}\| M_{v}(L)=m_{v}(L), \quad \text { or } \quad \dot{\omega}_{v}(L)=0, \\
-\|\boldsymbol{t}\| M_{v}(0)=m_{v}(0), \quad \text { or } \quad \dot{\omega}_{\nu}(0)=0 .
\end{gathered}
$$

\section{Tangent equilibrium operator}

As illustrated in [58], the tangent stiffness operator is defined as the second (covariant) gradient (the Hessian) of the internal energy on the tangent space to the generic configuration of the rod. In general, it is not easy to define a parallel transport operator on the state space, but if it is introduced a Riemmanian's metric by means of the Kozsul formula, it is possible to define the Levi-Civita connection for the configuration space induced by the assumed metric. We stress that the Gateaux derivatives does not represent the components of the (covariant) gradient in the tangent space of the structural model, i.e., the Gateaux derivative is not a tensor and plays the role of the partial derivative in the Riemann's geometry.

\subsection{Second Gateaux derivative: loss of symmetry}

In order to show the noncommutative nature of the second Gateaux derivative, in this section we derive the second Gateaux derivatives of the vectors of the intrinsic triad, which turn out to be nonsymmetric. As it will be evident from the results obtained, the cause of the nonsymmetry stands in the noncommutativity of the $\boldsymbol{\Lambda}$ rotation operator. This observation will be of great importance in the subsequent developments. 
Let $\bar{\varphi}$ and $\dot{\varphi}$ be any two elements of $\mathrm{T}_{\varphi} \mathbb{M}$. Then, the second Gateaux derivatives of the cross section directors are given by

$$
\begin{aligned}
\partial_{\bar{\varphi}} \partial_{\dot{\varphi}} \hat{t}(\varphi) & =\partial_{\dot{\varphi}} \partial_{\bar{\varphi}} \hat{\boldsymbol{t}}(\varphi), \\
\partial_{\bar{\varphi}} \partial_{\dot{\varphi}} \hat{\boldsymbol{n}}(\varphi) & \neq \partial_{\dot{\varphi}} \partial_{\bar{\varphi}} \hat{\boldsymbol{n}}(\varphi), \\
\partial_{\bar{\varphi}} \partial_{\dot{\varphi}} \hat{\boldsymbol{v}}(\varphi) & \neq \partial_{\dot{\varphi}} \partial_{\bar{\varphi}} \hat{\boldsymbol{v}}(\varphi),
\end{aligned}
$$

where, clearly, symmetry holds only on for the second directional derivative of the unit tangent vector. In particular, the second Gateaux derivative of the unit vector $\hat{\boldsymbol{n}}$ splits in

$$
\partial_{\bar{\varphi}} \partial_{\dot{\varphi}} \hat{\boldsymbol{n}}(\varphi)=\left(\partial_{\overline{\boldsymbol{u}}} \partial_{\dot{\boldsymbol{u}}}+\partial_{\overline{\boldsymbol{u}}} \partial_{\dot{\phi}}+\partial_{\bar{\phi}} \partial_{\dot{\boldsymbol{u}}}+\partial_{\bar{\phi}} \partial_{\dot{\phi}}\right) \hat{\boldsymbol{n}}(\varphi)
$$

where the first addend is given by

$$
\begin{aligned}
\partial_{\overline{\boldsymbol{u}}} \partial_{\dot{\boldsymbol{u}}}(\hat{\boldsymbol{n}}(\varphi))= & {\left[\frac{\mathrm{d} \overline{\boldsymbol{u}}}{\mathrm{d} S} \cdot\left(\frac{\hat{\boldsymbol{t}} \otimes \hat{\boldsymbol{n}}}{\|\boldsymbol{t}\|^{2}}+\frac{\hat{\boldsymbol{n}} \otimes \hat{\boldsymbol{t}}}{\|\boldsymbol{t}\|^{2}}\right) \cdot \frac{\mathrm{d} \dot{\boldsymbol{u}}}{\mathrm{d} S}\right] \hat{\boldsymbol{t}}+} \\
& -\left(\frac{\mathrm{d} \overline{\boldsymbol{u}}}{\mathrm{d} S} \cdot \frac{\hat{\boldsymbol{n}} \otimes \hat{\boldsymbol{n}}}{\|\boldsymbol{t}\|^{2}} \cdot \frac{\mathrm{d} \dot{\boldsymbol{u}}}{\mathrm{d} S}\right) \hat{\boldsymbol{n}}+ \\
& -\left(\frac{\mathrm{d} \overline{\boldsymbol{u}}}{\mathrm{d} S} \cdot \frac{\hat{\boldsymbol{v}} \otimes \hat{\boldsymbol{n}}}{\|\boldsymbol{t}\|^{2}} \cdot \frac{\mathrm{d} \dot{\boldsymbol{u}}}{\mathrm{d} S}\right) \hat{\boldsymbol{v}}
\end{aligned}
$$

From the Eq. (35), it appears that the components along the vector $\hat{\boldsymbol{t}}$ and $\hat{\boldsymbol{n}}$ are symmetric, while the component along $\hat{v}$ is nonsymmetric. The remaining terms of the right-hand side of the Eq. (35) are given by

$$
\begin{aligned}
\partial_{\overline{\boldsymbol{u}}} \partial_{\dot{\phi}}(\hat{\boldsymbol{n}}(\varphi)) & =-\frac{\dot{\phi}}{\|\boldsymbol{t}\|}\left(\frac{\mathrm{d} \overline{\boldsymbol{u}}}{\mathrm{d} S} \cdot \hat{\boldsymbol{v}}\right) \hat{\boldsymbol{t}}, \\
\partial_{\bar{\phi}} \partial_{\dot{\boldsymbol{u}}}(\hat{\boldsymbol{n}}(\boldsymbol{\varphi})) & =-\frac{\bar{\phi}}{\|\boldsymbol{t}\|}\left(\frac{\mathrm{d} \dot{\boldsymbol{u}}}{\mathrm{d} S} \cdot \hat{\boldsymbol{v}}\right) \hat{\boldsymbol{t}}, \\
\partial_{\bar{\phi}} \partial_{\dot{\phi}}(\hat{\boldsymbol{n}}(\boldsymbol{\varphi})) & =-\bar{\phi} \dot{\phi} \hat{\boldsymbol{n}},
\end{aligned}
$$

and are symmetric.

The second Gateaux derivative of the unit vector $\hat{\mathbf{v}}$ splits in

$$
\partial_{\bar{\varphi}} \partial_{\dot{\varphi}} \hat{v}(\varphi)=\left(\partial_{\bar{u}} \partial_{\dot{\boldsymbol{u}}}+\partial_{\overline{\boldsymbol{u}}} \partial_{\dot{\phi}}+\partial_{\bar{\phi}} \partial_{\dot{\boldsymbol{u}}}+\partial_{\bar{\phi}} \partial_{\dot{\phi}}\right) \hat{\boldsymbol{v}}(\varphi)
$$

where the first term is given by

$$
\begin{aligned}
\partial_{\overline{\boldsymbol{u}}} \partial_{\dot{\boldsymbol{u}}}(\hat{\boldsymbol{v}}(\varphi))= & \left.\frac{\mathrm{d} \overline{\boldsymbol{u}}}{\mathrm{d} S} \cdot\left(\frac{\hat{\boldsymbol{t}} \otimes \hat{\boldsymbol{v}}}{\|\boldsymbol{t}\|^{2}}+\frac{\hat{\boldsymbol{v}} \otimes \hat{\boldsymbol{t}}}{\|\boldsymbol{t}\|^{2}}\right) \cdot \frac{\mathrm{d} \dot{\boldsymbol{u}}}{\mathrm{d} S}\right] \hat{\boldsymbol{t}}+ \\
& -\left(\frac{\mathrm{d} \overline{\boldsymbol{u}}}{\mathrm{d} S} \cdot \frac{\hat{\boldsymbol{n}} \otimes \hat{\boldsymbol{v}}}{\|\boldsymbol{t}\|^{2}} \cdot \frac{\mathrm{d} \dot{\boldsymbol{u}}}{\mathrm{d} S}\right) \hat{\boldsymbol{n}}+ \\
& -\left(\frac{\mathrm{d} \overline{\boldsymbol{u}}}{\mathrm{d} S} \cdot \frac{\hat{\boldsymbol{v}} \otimes \hat{\boldsymbol{v}}}{\|\boldsymbol{t}\|^{2}} \cdot \frac{\mathrm{d} \dot{\boldsymbol{u}}}{\mathrm{d} S}\right) \hat{\boldsymbol{v}}
\end{aligned}
$$

From the Eq. (38), it appears that the components along $\hat{\boldsymbol{t}}$ and $\hat{\boldsymbol{v}}$ are symmetric, while the component along $\hat{\boldsymbol{n}}$ is nonsymmetric. The remaining terms of the right-hand side of the Eq. (38) are given by

$$
\begin{aligned}
\partial_{\overline{\boldsymbol{u}}} \partial_{\dot{\phi}}(\hat{\boldsymbol{v}}(\varphi)) & =\frac{\dot{\phi}}{\|\boldsymbol{t}\|}\left(\frac{\mathrm{d} \overline{\boldsymbol{u}}}{\mathrm{d} S} \cdot \hat{\boldsymbol{n}}\right) \hat{\boldsymbol{t}}, \\
\partial_{\bar{\phi}} \partial_{\dot{\boldsymbol{u}}}(\hat{\boldsymbol{v}}(\varphi)) & =\frac{\bar{\phi}}{\|\boldsymbol{t}\|}\left(\frac{\mathrm{d} \dot{\boldsymbol{u}}}{\mathrm{d} S} \cdot \hat{\boldsymbol{n}}\right) \hat{\boldsymbol{t}}, \\
\partial_{\bar{\phi}} \partial_{\dot{\phi}}(\hat{\boldsymbol{v}}(\varphi)) & =-\bar{\phi} \dot{\phi} \hat{\boldsymbol{v}},
\end{aligned}
$$

and are symmetric. 
In the following sections, we define the connection for the tangent space to the configuration space of the structural model considered, i.e., we define the connection for the Kirchhoff rod model; in particular, we adopt the unique torsion-free connection compatible with the assumed metric.

\subsection{The Koszul formula: the Levi-Civita connection}

Let $\left\{\mathbb{M}, \mathbb{G}_{\varphi}\right\}$ be a Riemannian manifold, equipped with the metric $\mathbb{G}_{\varphi}: \mathrm{T}_{\varphi} \mathbb{M} \times \mathrm{T}_{\varphi} \mathbb{M} \rightarrow \mathbb{R}$. The fundamental theorem of Riemannian geometry states that there is a unique connection, $\nabla$, (the Levi-Civita connection), which preserves the metric tensor, that is, such that for $\dot{\varphi}, \bar{\varphi}, \overline{\bar{\varphi}} \in \mathrm{T}_{\varphi} \mathbb{M}$,

$$
\nabla_{\bar{\varphi}} \mathbb{G}_{\varphi}(\dot{\varphi}, \overline{\bar{\varphi}})=\mathbb{G}_{\varphi}\left(\nabla_{\bar{\varphi}} \dot{\varphi}, \overline{\bar{\varphi}}\right)+\mathbb{G}_{\varphi}\left(\dot{\varphi}, \nabla_{\bar{\varphi}} \overline{\bar{\varphi}}\right), \quad \forall \dot{\varphi}, \bar{\varphi}, \overline{\bar{\varphi}}, \in \mathrm{T}_{\varphi} \mathbb{M},
$$

and that is torsion-free, i.e., for all differentiable $f: \mathbb{M} \rightarrow \mathbb{R}$

$$
[\bar{\varphi}, \dot{\varphi}] f=\nabla_{\bar{\varphi}} \dot{\varphi} f-\nabla_{\dot{\varphi}} \bar{\varphi} f, \quad \forall \dot{\varphi}, \bar{\varphi}, \in \mathrm{T}_{\varphi} \mathbb{M} .
$$

The unique Levi-Civita connection can be obtained by the Koszul formula, i.e., $\forall \dot{\varphi}, \overline{\bar{\varphi}}, \bar{\varphi} \in \mathrm{T}_{\varphi} \mathbb{M}$

$$
\begin{aligned}
2 \mathbb{G}_{\varphi}\left(\nabla_{\bar{\varphi}} \dot{\varphi}, \overline{\bar{\varphi}}\right)= & \partial_{\bar{\varphi}} \mathbb{G}_{\varphi}(\dot{\boldsymbol{\varphi}}, \overline{\bar{\varphi}})+\partial_{\dot{\varphi}} \mathbb{G}_{\varphi}(\bar{\varphi}, \overline{\bar{\varphi}})-\partial_{\overline{\bar{\varphi}}} \mathbb{G}_{\varphi}(\dot{\boldsymbol{\varphi}}, \bar{\varphi})+ \\
& +\mathbb{G}_{\varphi}([\overline{\bar{\varphi}}, \dot{\varphi}], \overline{\bar{\varphi}})-\mathbb{G}_{\varphi}([\dot{\varphi}, \overline{\bar{\varphi}}], \bar{\varphi})-\mathbb{G}_{\varphi}([\bar{\varphi}, \overline{\bar{\varphi}}], \dot{\varphi}) .
\end{aligned}
$$

Now, we particularize the previous formulae to the Kirchhoff rod model presented. We adopt the following metric:

$$
\mathbb{G}_{\varphi}(\overline{\boldsymbol{\varphi}}, \dot{\boldsymbol{\varphi}}):=\int_{0}^{L}(\overline{\boldsymbol{u}} \cdot \dot{\boldsymbol{u}}+\overline{\boldsymbol{\omega}}(\overline{\boldsymbol{u}}) \cdot \dot{\boldsymbol{\omega}}(\dot{\boldsymbol{u}})+\bar{\phi} \dot{\phi}) \mathrm{d} S .
$$

The choice is motivated by the fact that any variation of the position of the centroid line implies a rotation of the directors.

Observing that the metric defined by the definition (43) does not depend explicitly on the configuration of the manifold $\varphi$, the first three terms on the right-hand side of the Koszul formula trivially vanish so that only the second line of Eq. (42) remains. It can be explicitly evaluated using the expression for the Lie parenthesis.

The Lie parenthesis is obtained evaluating the commutator for two independent elements of $\mathrm{T}_{\varphi} \mathbb{M}$. Recall that to the variation $\tilde{\boldsymbol{u}}$ is associated a translation and a rotation of the directors represented by a skew-symmetric matrix (an infinitesimal rotation superposed to $\boldsymbol{\Lambda}$ ). The translation commutes with any translation and rotation, so that, indicating by $\boldsymbol{\Omega}$ the skew-symmetric matrix associated with any rotation vector $\boldsymbol{\omega}_{b}$ and with $\boldsymbol{\Phi}$ the skew-symmetric matrix associated with any rotation $\phi \hat{\boldsymbol{t}}$ the commutator reduces to the following:

$$
[\bar{\varphi}, \dot{\varphi}]=\operatorname{axial}[\bar{\Omega} \dot{\Omega}+\overline{\boldsymbol{\Phi}} \dot{\boldsymbol{\Phi}}-\dot{\boldsymbol{\Omega}} \bar{\Omega}-\overline{\boldsymbol{\Phi}} \dot{\boldsymbol{\Phi}}]=\bar{\omega}_{b} \times \dot{\omega}_{b}
$$

Using this result in Koszul's formula (42), and observing that the second and third terms of the second line of the expression vanish for the properties of the triple product, we have

$$
2 \mathbb{G}_{\varphi}\left(\nabla_{\bar{\varphi}} \dot{\varphi}, \overline{\bar{\varphi}}\right)=\left[\bar{\omega}_{b}, \dot{\omega}_{b}\right] \cdot \overline{\bar{\omega}}_{b}=\overline{\boldsymbol{\omega}}_{b} \times \dot{\omega}_{b} \cdot \overline{\bar{\omega}}_{b} .
$$

Therefore, the only non-null structure coefficient is

$$
\begin{aligned}
\nabla_{\overline{\boldsymbol{\omega}}_{b}} \dot{\boldsymbol{\omega}}_{b} & :=\frac{1}{2}\left[\overline{\boldsymbol{\omega}}_{b}, \dot{\boldsymbol{\omega}}_{b}\right]=\frac{1}{2}\left(\overline{\boldsymbol{\omega}}_{b} \times \dot{\boldsymbol{\omega}}_{b}\right) \\
& =\left(\frac{\bar{\omega}_{n} \dot{\omega}_{\nu}-\bar{\omega}_{\nu} \dot{\omega}_{n}}{2}\right) \hat{\boldsymbol{t}} .
\end{aligned}
$$




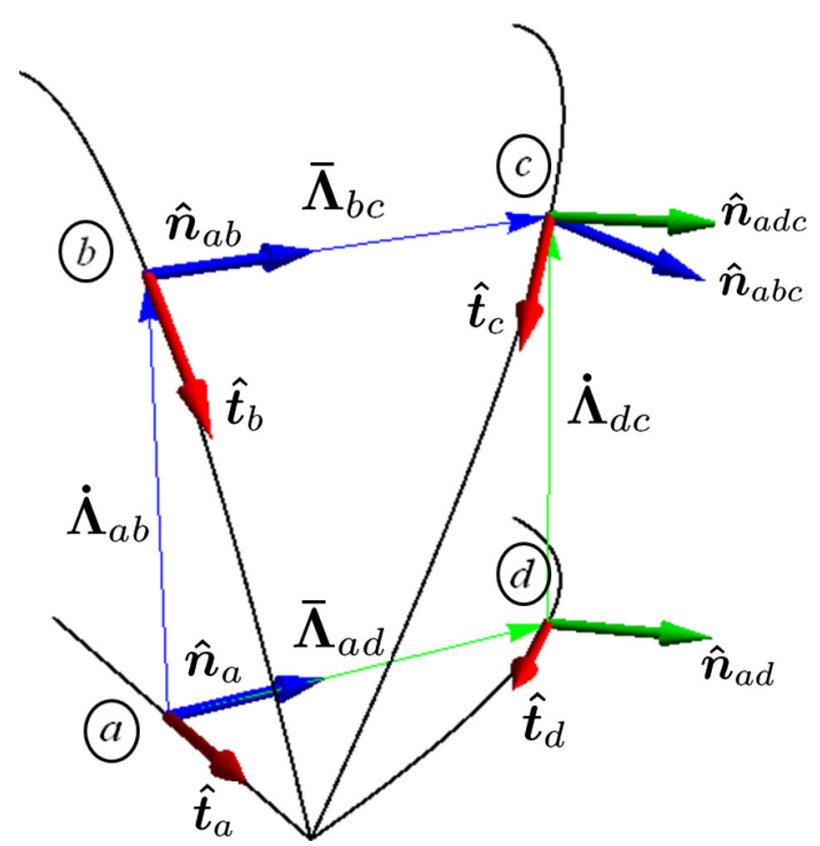

Fig. 1 Graphical interpretation of the nonvanishing connection symbol, corresponding to a rotation around the current unit tangent vector $\hat{\boldsymbol{t}}$ (a torsional rotation)

Substituting the relations $\dot{\omega}_{\nu}=\frac{1}{\|t\|}\left(\frac{\mathrm{d} \dot{\boldsymbol{u}}}{\mathrm{d} S} \cdot \hat{\boldsymbol{n}}\right)$ and $\dot{\omega}_{n}=-\frac{1}{\|\boldsymbol{t}\|}\left(\frac{\mathrm{d} \dot{\boldsymbol{u}}}{\mathrm{d} S} \cdot \hat{\boldsymbol{v}}\right)$, that hold for the Kirchhoff assumptions, the connection coefficient (46) takes the form:

$$
\begin{aligned}
\hat{\boldsymbol{t}} \cdot \nabla_{\overline{\boldsymbol{\omega}}_{b}} \dot{\boldsymbol{\omega}}_{b} & =\frac{1}{2}\left[\left(\frac{1}{\|\boldsymbol{t}\|} \frac{\mathrm{d} \overline{\boldsymbol{u}}}{\mathrm{d} S} \cdot \hat{\boldsymbol{n}}\right)\left(\frac{1}{\|\boldsymbol{t}\|} \frac{\mathrm{d} \dot{\boldsymbol{u}}}{\mathrm{d} S} \cdot \hat{\boldsymbol{v}}\right)-\left(\frac{1}{\|\boldsymbol{t}\|} \frac{\mathrm{d} \dot{\boldsymbol{u}}}{\mathrm{d} S} \cdot \hat{\boldsymbol{n}}\right)\left(\frac{1}{\|\boldsymbol{t}\|} \frac{\mathrm{d} \overline{\boldsymbol{u}}}{\mathrm{d} S} \cdot \hat{\boldsymbol{v}}\right)\right], \\
& =\frac{1}{2}\left[\frac{\mathrm{d} \overline{\boldsymbol{u}}}{\mathrm{d} S} \cdot \frac{\hat{\boldsymbol{n}} \otimes \hat{\boldsymbol{v}}}{\|\boldsymbol{t}\|^{2}} \cdot \frac{\mathrm{d} \dot{\boldsymbol{u}}}{\mathrm{d} S}-\frac{\mathrm{d} \dot{\boldsymbol{u}}}{\mathrm{d} S} \cdot \frac{\hat{\boldsymbol{n}} \otimes \hat{\boldsymbol{v}}}{\|\boldsymbol{t}\|^{2}} \cdot \frac{\mathrm{d} \overline{\boldsymbol{u}}}{\mathrm{d} S}\right] .
\end{aligned}
$$

Applying the vector (46) to the unit triad $\{\hat{\boldsymbol{t}}, \hat{\boldsymbol{n}}, \hat{\boldsymbol{v}}\}$, we get the skew-symmetric part of the second variation of the unit triad that, as evidenced by Eqs. (35),(38), is associated with the bending rotations only. This fact is graphically represented in Fig. 1 that shows two subsequent bending rotations around two orthogonal axes occurring in reversed order $a \rightarrow b \rightarrow c$ and $a \rightarrow d \rightarrow c$. The red arrow denotes the tangent to the rod axis, and the blue or green arrow denotes the normal vector. The final configurations differ only by a torsional rotation.

\subsection{Second covariant gradient: the Hessian}

We are now in position for evaluating the second covariant gradient of the internal energy potential $\psi_{\text {int }}(\varphi)$. Differentiating the first gradient of $\psi_{\text {int }}$, given by

$$
\nabla(\psi)=\partial_{\dot{\varphi}}(\psi(\varphi)) \otimes \dot{\varphi}^{\natural}
$$

we have the following:

$$
\nabla^{2}(\psi)=\partial_{\bar{\varphi}} \partial_{\dot{\boldsymbol{\varphi}}}(\psi(\boldsymbol{\varphi})) \otimes \dot{\boldsymbol{\varphi}}^{\natural} \otimes \overline{\boldsymbol{\varphi}}^{\natural}+\partial_{\dot{\boldsymbol{\varphi}}}(\psi(\boldsymbol{\varphi})) \otimes \nabla_{\bar{\varphi}} \dot{\boldsymbol{\varphi}}^{\natural} \otimes \overline{\boldsymbol{\varphi}}^{\natural},
$$

where the apex $\downarrow$ indicates the contravariant basis. With simple algebra, we get:

$$
\begin{aligned}
\nabla^{2}(\psi) & =\left[\partial_{\bar{\varphi}} \partial_{\dot{\varphi}}(\psi(\boldsymbol{\varphi}))-\partial_{\dot{\varphi}}(\psi(\boldsymbol{\varphi}))\left(\nabla_{\bar{\varphi}} \dot{\boldsymbol{\varphi}} \cdot \dot{\boldsymbol{\varphi}}^{\natural}\right)\right] \otimes \dot{\boldsymbol{\varphi}}^{\natural} \otimes \overline{\boldsymbol{\varphi}}^{\natural} \\
& =\left[\partial_{\bar{\varphi}} \partial_{\dot{\varphi}}(\psi(\boldsymbol{\varphi}))-\partial_{\nabla_{\bar{\varphi}} \dot{\varphi}}(\psi(\boldsymbol{\varphi}))\right] \otimes \dot{\boldsymbol{\varphi}}^{\natural} \otimes \overline{\boldsymbol{\varphi}}^{\natural} .
\end{aligned}
$$


The first term in Eq. (50) is the second directional Gateaux derivative of the energy functional. It splits in the sum of two parts, the first known as the tangent material-stiffness, $\mathbb{K}_{M}$ symmetric for an associated material model, and the second known as the tangent geometric-stiffness, $\mathbb{G}$ that turns out to be nonsymmetric, if the tangent map is noncommutative. In [58], Simo demonstrated that splitting the geometric stiffness into its symmetric and skew-symmetric parts, the latter is equal to the opposite of the second term of the result (50), restoring the symmetry of the second covariant derivative. He proved this property for a Timoshenko rod, using the $\mathrm{SO}$ (3) group for the rotations. In this work, we prove that the same conclusion is also true for the configuration space introduced in (12), valid for a Kirchhoff-Love rod, with the metric (43).

With an obvious meaning of the symbols, the tangent stiffness operator, from expression (50), is written as

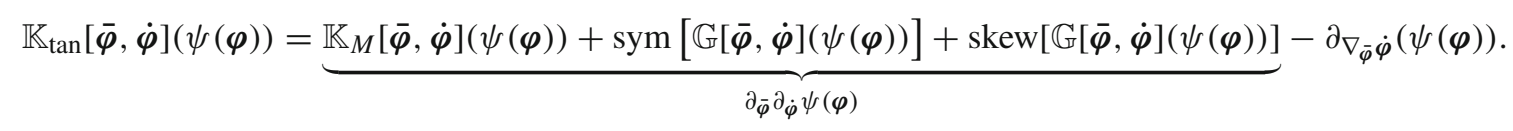

Having obtained the coefficients of structure, we are able to evaluate the last term in (51). From the property that the connection is torsion-free, it will be found that $\operatorname{skew}[\mathbb{G}[\overline{\boldsymbol{\varphi}}, \dot{\boldsymbol{\varphi}}](\psi(\varphi))]-\partial_{\nabla_{\bar{\varphi}} \dot{\varphi}}(\psi(\boldsymbol{\varphi}))=\mathbf{0}$, so that the tangent stiffness operator reduces to

$$
\mathbb{K}_{\tan }[\overline{\boldsymbol{\varphi}}, \dot{\boldsymbol{\varphi}}](\psi(\boldsymbol{\varphi}))=\mathbb{K}_{M}[\overline{\boldsymbol{\varphi}}, \dot{\boldsymbol{\varphi}}](\psi(\boldsymbol{\varphi}))+\operatorname{sym}[\mathbb{G}[\overline{\boldsymbol{\varphi}}, \dot{\boldsymbol{\varphi}}](\psi(\boldsymbol{\varphi}))] .
$$

\subsection{Material tangent stiffness: $\mathbb{K}_{M}$}

The material tangent stiffness can be evaluated as

$$
\begin{aligned}
\mathbb{M}[\overline{\boldsymbol{\varphi}}, \dot{\boldsymbol{\varphi}}](\psi(\varphi))= & \int_{0}^{L} E A\left(\partial_{\bar{\varphi}} \varepsilon(\varphi)\right)\left(\partial_{\dot{\varphi}} \varepsilon(\varphi)\right) \mathrm{d} S+\int_{0}^{L} E I_{n}\left(\partial_{\bar{\varphi}} \chi_{n}(\varphi)\right)\left(\partial_{\dot{\varphi}} \chi_{n}(\varphi)\right) \mathrm{d} S+ \\
& +\int_{0}^{L} E I_{v}\left(\partial_{\bar{\varphi}} \chi_{v}(\varphi)\right)\left(\partial_{\dot{\varphi}} \chi_{v}(\boldsymbol{\varphi})\right) \mathrm{d} S+\int_{0}^{L} G J_{t}\left(\partial_{\bar{\varphi}} \chi_{t}(\varphi)\right)\left(\partial_{\dot{\varphi}} \chi_{t}(\boldsymbol{\varphi})\right) \mathrm{d} S .
\end{aligned}
$$

Using the results (24) for the variations of the strain measures, we have the following: material axial stiffness

$$
\mathbb{K}_{M}^{\mathrm{ax}}[\overline{\boldsymbol{\varphi}}, \dot{\boldsymbol{\varphi}}](\psi(\boldsymbol{\varphi}))=\int_{0}^{L} E A\left(\frac{\mathrm{d} \overline{\boldsymbol{u}}}{\mathrm{d} S} \cdot \boldsymbol{t} \otimes \boldsymbol{t} \cdot \frac{\mathrm{d} \dot{\boldsymbol{u}}}{\mathrm{d} S}\right) \mathrm{d} S
$$

material bending stiffnesses

$$
\mathbb{K}_{M}^{v}[\overline{\boldsymbol{\varphi}}, \dot{\boldsymbol{\varphi}}](\psi(\varphi))=\int_{0}^{L} E I_{v}\left[\left(\frac{d^{2} \overline{\boldsymbol{u}}}{\mathrm{d} S^{2}}-\bar{\phi} \boldsymbol{b}\right) \cdot \hat{\boldsymbol{n}} \otimes \hat{\boldsymbol{n}} \cdot\left(\frac{d^{2} \dot{\boldsymbol{u}}}{\mathrm{d} S^{2}}-\dot{\phi} \boldsymbol{b}\right)\right] \mathrm{d} S,
$$

and

$$
\mathbb{K}_{M}^{\mathrm{n}}[\overline{\boldsymbol{\varphi}}, \dot{\boldsymbol{\varphi}}](\psi(\boldsymbol{\varphi}))=\int_{0}^{L} E I_{n}\left[\left(-\frac{d^{2} \overline{\boldsymbol{u}}}{\mathrm{d} S^{2}}+\bar{\phi} \boldsymbol{b}\right) \cdot \hat{\boldsymbol{v}} \otimes \hat{\boldsymbol{v}} \cdot\left(-\frac{d^{2} \dot{\boldsymbol{u}}}{\mathrm{d} S^{2}}+\dot{\phi} \boldsymbol{b}\right)\right] \mathrm{d} S .
$$

material torsion stiffness

$$
\mathbb{K}_{M}^{\mathrm{t}}[\overline{\boldsymbol{\varphi}}, \dot{\boldsymbol{\varphi}}](\psi(\boldsymbol{\varphi}))=\int_{0}^{L} G J_{t}\left(\frac{\mathrm{d} \bar{\phi}}{\mathrm{d} S}+\frac{1}{\|\boldsymbol{t}\|^{2}} \frac{\mathrm{d} \overline{\boldsymbol{u}}}{\mathrm{d} S} \cdot \boldsymbol{b}\right)\left(\frac{\mathrm{d} \dot{\phi}}{\mathrm{d} S}+\frac{1}{\|\boldsymbol{t}\|^{2}} \frac{\mathrm{d} \dot{\boldsymbol{u}}}{\mathrm{d} S} \cdot \boldsymbol{b}\right) \mathrm{d} S .
$$

In the definitions (54), (55), (56), and (57), the vectors $\boldsymbol{t}, \boldsymbol{n}, \boldsymbol{v}$, and $\boldsymbol{b}$ are referred to the current configuration $\varphi$. 
The formal expression of the geometric tangent stiffness is

$$
\begin{aligned}
\mathbb{G}[\bar{\varphi}, \dot{\varphi}](\psi(\varphi))= & \int_{0}^{L} E A \varepsilon(\varphi)\left(\partial_{\bar{\varphi}} \partial_{\dot{\varphi}} \varepsilon(\varphi)\right) \mathrm{d} S+\int_{0}^{L} E I_{n} \chi_{n}(\varphi)\left(\partial_{\bar{\varphi}} \partial_{\dot{\varphi}} \chi_{n}(\varphi)\right) \mathrm{d} S+ \\
& +\int_{0}^{L} E I_{\nu} \chi_{\nu}(\varphi)\left(\partial_{\bar{\varphi}} \partial_{\dot{\varphi}} \chi_{\nu}(\varphi)\right) \mathrm{d} S+\int_{0}^{L} G J_{t} \chi_{t}(\varphi)\left(\partial_{\bar{\varphi}} \partial_{\dot{\varphi}} \chi_{t}(\varphi)\right) \mathrm{d} S .
\end{aligned}
$$

Preliminarily, we explicitly calculate the second Gateaux directional derivative of the strain measures that appear in the formula (58), starting from the first variations stated in Eq. (24) that are recalled here for convenience in a slightly more elaborate form, for an arbitrary element $\{\dot{\boldsymbol{u}}, \dot{\phi}\} \in \mathrm{T}_{\varphi} \mathbb{M}$ :

$$
\begin{aligned}
\partial_{\dot{\varphi}} \varepsilon(\varphi) & =\frac{\mathrm{d} \dot{\boldsymbol{u}}}{\mathrm{d} S} \cdot \boldsymbol{t} \\
-\partial_{\dot{\varphi}} \chi_{v}(\varphi) & =\left[-\frac{d^{2} \dot{\boldsymbol{u}}}{\mathrm{d} S^{2}}+\dot{\phi}\left(\hat{\boldsymbol{t}} \times \frac{\mathrm{d} \boldsymbol{t}}{\mathrm{d} S}\right)\right] \cdot \hat{\boldsymbol{n}} \\
& =\left[-\frac{d}{\mathrm{~d} S}\left(\frac{\mathrm{d} \dot{\boldsymbol{u}}}{\mathrm{d} S}\right)+\frac{1}{\|\boldsymbol{t}\|} \frac{\mathrm{d}\|\boldsymbol{t}\|}{\mathrm{d} S} \frac{\mathrm{d} \dot{\boldsymbol{u}}}{\mathrm{d} S}+\dot{\phi}\left(\hat{\boldsymbol{t}} \times \frac{\mathrm{d} \boldsymbol{t}}{\mathrm{d} S}\right)\right] \cdot \hat{\boldsymbol{n}}, \\
\partial_{\dot{\varphi}} \chi_{n}(\varphi) & =\left[-\frac{d^{2} \dot{\boldsymbol{u}}}{\mathrm{d} S^{2}}+\dot{\phi}\left(\hat{\boldsymbol{t}} \times \frac{\mathrm{d} \boldsymbol{t}}{\mathrm{d} S}\right)\right] \cdot \hat{\boldsymbol{v}} \\
& =\left[-\frac{d}{\mathrm{~d} S}\left(\frac{\mathrm{d} \dot{\boldsymbol{u}}}{\mathrm{d} S}\right)+\frac{1}{\|\boldsymbol{t}\|} \frac{\mathrm{d}\|\boldsymbol{t}\|}{\mathrm{d} S} \frac{\mathrm{d} \dot{\boldsymbol{u}}}{\mathrm{d} S}+\dot{\phi}\left(\hat{\boldsymbol{t}} \times \frac{\mathrm{d} \boldsymbol{t}}{\mathrm{d} S}\right)\right] \cdot \hat{\boldsymbol{v}}, \\
\partial_{\dot{\varphi}} \chi_{t}(\varphi) & =\frac{\mathrm{d} \dot{\phi}}{\mathrm{d} S}+\frac{1}{\|\boldsymbol{t}\|} \frac{\mathrm{d} \dot{\boldsymbol{u}}}{\mathrm{d} S} \cdot\left(\hat{\boldsymbol{t}} \times \frac{\mathrm{d} \hat{\boldsymbol{t}}}{\mathrm{d} S}\right) .
\end{aligned}
$$

\subsubsection{Second Gateaux derivative of $\varepsilon$}

The second derivative of the axial strain-rate measures is given by

$$
\partial_{\bar{\varphi}} \partial_{\dot{\varphi}} \varepsilon(\varphi)=\frac{\mathrm{d} \dot{\boldsymbol{u}}}{\mathrm{d} S} \cdot \frac{\mathrm{d} \overline{\boldsymbol{u}}}{\mathrm{d} S}
$$

and this is trivially symmetric.

\subsubsection{Second Gateaux derivative of $\chi_{v}$}

The calculation of the second Gateaux derivative of $\chi_{v}((60))$ splits in two parts,

$$
\partial_{\bar{\varphi}} \partial_{\dot{\varphi}} \chi_{v}(\varphi)=\partial_{\bar{u}} \partial_{\dot{\varphi}} \chi_{v}(\varphi)+\partial_{\bar{\phi}} \partial_{\dot{\varphi}} \chi_{v}(\varphi)
$$

where the first defines the action of the variation $\overline{\boldsymbol{u}}$ only, while the second defines the action of the variation of the rotation $\bar{\phi}$ on the strain-rate measure (60) along the generic tangent direction $\bar{\varphi} \in \mathrm{T}_{\varphi} \mathbb{M}$. In particular, one has the following:

$$
\begin{aligned}
\partial_{\overline{\boldsymbol{u}}} \partial_{\dot{\varphi}} \chi_{v}(\boldsymbol{\varphi})= & -\frac{d^{2} \dot{\boldsymbol{u}}}{\mathrm{d} S^{2}} \cdot \frac{\hat{\boldsymbol{t}} \otimes \hat{\boldsymbol{n}}}{\|\boldsymbol{t}\|} \cdot \frac{\mathrm{d} \overline{\boldsymbol{u}}}{\mathrm{d} S}-\frac{\mathrm{d} \dot{\boldsymbol{u}}}{\mathrm{d} S} \cdot \frac{\hat{\boldsymbol{n}} \otimes \hat{\boldsymbol{t}}}{\|\boldsymbol{t}\|} \cdot \frac{d^{2} \overline{\boldsymbol{u}}}{\mathrm{d} S^{2}}+ \\
& +\left(\frac{\mathrm{d} \hat{\boldsymbol{n}}}{\mathrm{d} S} \cdot \hat{\boldsymbol{t}}\right) \frac{\mathrm{d} \overline{\boldsymbol{u}}}{\mathrm{d} S} \cdot \frac{\hat{\boldsymbol{n}} \otimes \hat{\boldsymbol{n}}}{\|\boldsymbol{t}\|} \cdot \frac{\mathrm{d} \dot{\boldsymbol{u}}}{\mathrm{d} S}+\left(\frac{\mathrm{d} \hat{\boldsymbol{v}}}{\mathrm{d} S} \cdot \hat{\boldsymbol{t}}\right) \frac{\mathrm{d} \overline{\boldsymbol{u}}}{\mathrm{d} S} \cdot \frac{\hat{\boldsymbol{v}} \otimes \hat{\boldsymbol{n}}}{\|\boldsymbol{t}\|} \cdot \frac{\mathrm{d} \dot{\boldsymbol{u}}}{\mathrm{d} S},
\end{aligned}
$$


and

$$
\partial_{\bar{\phi}} \partial_{\dot{\varphi}} \chi_{v}(\varphi)=\bar{\phi}\left(\hat{\boldsymbol{v}} \cdot \frac{d^{2} \dot{\boldsymbol{u}}}{\mathrm{d} S^{2}}\right)+\dot{\phi}\left(\hat{\boldsymbol{v}} \cdot \frac{d^{2} \overline{\boldsymbol{u}}}{\mathrm{d} S^{2}}\right)+\dot{\phi} \bar{\phi}\left(\frac{\mathrm{d} \hat{\boldsymbol{n}}}{\mathrm{d} S} \cdot \boldsymbol{t}\right)
$$

Expression (65) is not symmetric, while the second term, given by (66), is symmetric in its arguments. The result (65) is split in its symmetric and in its skew-symmetric parts:

$$
\partial_{\bar{u}} \partial_{\dot{\varphi}} \chi_{v}(\varphi)=\operatorname{sym}\left\{\partial_{\bar{u}} \partial_{\dot{\varphi}} \chi_{v}(\varphi)\right\}+\operatorname{skew}\left\{\partial_{\bar{u}} \partial_{\dot{\varphi}} \chi_{v}(\varphi)\right\}
$$

where the symmetric part is given by the sum of the first three terms in the right-hand side of Eq. (65), while the skew-symmetric part is defined by

$$
\begin{aligned}
\text { skew }\left\{\partial_{\overline{\boldsymbol{u}}} \partial_{\dot{\varphi}} \chi_{\nu}(\boldsymbol{\varphi})\right\} & =\left(\frac{\mathrm{d} \hat{\boldsymbol{v}}}{\mathrm{d} S} \cdot \boldsymbol{t}\right) \frac{1}{2}\left[\frac{\mathrm{d} \overline{\boldsymbol{u}}}{\mathrm{d} S} \cdot \frac{\hat{\boldsymbol{v}} \otimes \hat{\boldsymbol{n}}}{\|\boldsymbol{t}\|^{2}} \cdot \frac{\mathrm{d} \dot{\boldsymbol{u}}}{\mathrm{d} S}-\frac{\mathrm{d} \dot{\boldsymbol{u}}}{\mathrm{d} S} \cdot \frac{\hat{\boldsymbol{v}} \otimes \hat{\boldsymbol{n}}}{\|\boldsymbol{t}\|^{2}} \cdot \frac{\mathrm{d} \overline{\boldsymbol{u}}}{\mathrm{d} S}+\right] \\
& =-\left(\frac{\mathrm{d} \hat{\boldsymbol{v}}}{\mathrm{d} S} \cdot \boldsymbol{t}\right) \hat{\boldsymbol{t}} \cdot \nabla_{\overline{\boldsymbol{\omega}}} \dot{\boldsymbol{\omega}} .
\end{aligned}
$$

\subsubsection{Second Gateaux derivative of $\chi_{n}$}

The second derivative of $\chi_{n}$ is evaluated in a manner analogous to the previous case:

$$
\partial_{\bar{\varphi}} \partial_{\dot{\varphi}} \chi_{n}(\varphi)=\partial_{\overline{\boldsymbol{u}}} \partial_{\dot{\varphi}} \chi_{n}(\varphi)+\partial_{\bar{\phi}} \partial_{\dot{\varphi}} \chi_{n}(\varphi)
$$

The two terms are respectively given by

$$
\begin{aligned}
\partial_{\overline{\boldsymbol{u}}} \partial_{\dot{\varphi}} \chi_{n}(\boldsymbol{\varphi})= & \frac{d^{2} \dot{\boldsymbol{u}}}{\mathrm{d} S^{2}} \cdot \frac{\hat{\boldsymbol{t}} \otimes \hat{\boldsymbol{v}}}{\|\boldsymbol{t}\|} \cdot \frac{\mathrm{d} \overline{\boldsymbol{u}}}{\mathrm{d} S}+\frac{\mathrm{d} \dot{\boldsymbol{u}}}{\mathrm{d} S} \cdot \frac{\hat{\boldsymbol{v}} \otimes \hat{\boldsymbol{t}}}{\|\boldsymbol{t}\|} \cdot \frac{\mathrm{d}^{2} \overline{\boldsymbol{u}}}{\mathrm{d} S^{2}}+ \\
& -\left(\frac{\mathrm{d} \hat{\boldsymbol{v}}}{\mathrm{d} S} \cdot \hat{\boldsymbol{t}}\right) \frac{\mathrm{d} \overline{\boldsymbol{u}}}{\mathrm{d} S} \cdot \frac{\hat{\boldsymbol{v}} \otimes \hat{\boldsymbol{v}}}{\|\boldsymbol{t}\|} \cdot \frac{\mathrm{d} \dot{\boldsymbol{u}}}{\mathrm{d} S}-\left(\frac{\mathrm{d} \hat{\boldsymbol{n}}}{\mathrm{d} S} \cdot \hat{\boldsymbol{t}}\right) \frac{\mathrm{d} \overline{\boldsymbol{u}}}{\mathrm{d} S} \cdot \frac{\hat{\boldsymbol{n}} \otimes \hat{\boldsymbol{v}} \mathrm{d} \dot{\boldsymbol{u}}}{\|\boldsymbol{t}\|} \frac{\mathrm{d} S}{}
\end{aligned}
$$

and

$$
\partial_{\bar{\phi}} \partial_{\dot{\varphi}} \chi_{n}(\varphi)=\bar{\phi}\left(\frac{d^{2} \dot{\boldsymbol{u}}}{\mathrm{d} S^{2}} \cdot \hat{\boldsymbol{n}}\right)+\dot{\phi}\left(\frac{d^{2} \overline{\boldsymbol{u}}}{\mathrm{d} S^{2}} \cdot \hat{\boldsymbol{n}}\right)-\dot{\phi} \bar{\phi}\left(\frac{\mathrm{d} \hat{\boldsymbol{v}}}{\mathrm{d} S} \cdot \boldsymbol{t}\right)
$$

As in the previous case, the second term (71) is symmetric in its arguments, while the first, given by (70), is not symmetric and is split in its symmetric and in its skew-symmetric parts as follows:

$$
\partial_{\overline{\boldsymbol{u}}} \partial_{\dot{\varphi}} \chi_{n}(\varphi)=\operatorname{sym}\left\{\partial_{\overline{\boldsymbol{u}}} \partial_{\dot{\varphi}} \chi_{n}(\varphi)\right\}+\operatorname{skew}\left\{\partial_{\overline{\boldsymbol{u}}} \partial_{\dot{\varphi}} \chi_{n}(\varphi)\right\},
$$

where the symmetric part is given by the sum of the first three terms of the result (70), while the skew-symmetric part is

$$
\text { skew } \begin{aligned}
\left\{\partial_{\overline{\boldsymbol{u}}} \partial_{\dot{\varphi}} \chi_{n}(\boldsymbol{\varphi})\right\} & =\left(\frac{\mathrm{d} \hat{\boldsymbol{n}}}{\mathrm{d} S} \cdot \boldsymbol{t}\right) \frac{1}{2}\left[-\frac{\mathrm{d} \overline{\boldsymbol{u}}}{\mathrm{d} S} \cdot \frac{\hat{\boldsymbol{n}} \otimes \hat{\boldsymbol{v}}}{\|\boldsymbol{t}\|^{2}} \cdot \frac{\mathrm{d} \dot{\boldsymbol{u}}}{\mathrm{d} S}+\frac{\mathrm{d} \dot{\boldsymbol{u}}}{\mathrm{d} S} \cdot \frac{\hat{\boldsymbol{n}} \otimes \hat{\boldsymbol{v}}}{\|\boldsymbol{t}\|^{2}} \cdot \frac{\mathrm{d} \overline{\boldsymbol{u}}}{\mathrm{d} S}\right] \\
& =-\left(\frac{\mathrm{d} \hat{\boldsymbol{n}}}{\mathrm{d} S} \cdot \boldsymbol{t}\right) \hat{\boldsymbol{t}} \cdot \nabla_{\overline{\boldsymbol{\omega}}} \dot{\boldsymbol{\omega}} .
\end{aligned}
$$


4.5.4 Second Gateaux derivative of $\chi_{t}$

Starting from (62), the second directional derivative of the torsion curvature is

$$
\partial_{\bar{\varphi}} \partial_{\dot{\varphi}} \chi_{t}(\varphi)=\partial_{\bar{u}} \partial_{\dot{\varphi}} \chi_{t}(\varphi), \quad \partial_{\bar{\phi}} \partial_{\dot{\varphi}} \chi_{t}(\varphi)=\mathbf{0} .
$$

where

$$
\begin{aligned}
\partial_{\overline{\boldsymbol{u}}} \partial_{\dot{\varphi}} \chi_{t}(\boldsymbol{\varphi})= & -\frac{1}{\|\boldsymbol{t}\|^{2}}\left(\frac{\mathrm{d} \hat{\boldsymbol{t}}}{\mathrm{d} S} \cdot \hat{\boldsymbol{n}}\right)\left\{\left(\frac{\mathrm{d} \overline{\boldsymbol{u}}}{\mathrm{d} S} \cdot \hat{\boldsymbol{t}}\right)\left(\frac{\mathrm{d} \dot{\boldsymbol{u}}}{\mathrm{d} S} \cdot \hat{\boldsymbol{v}}\right)+\left(\frac{\mathrm{d} \dot{\boldsymbol{u}}}{\mathrm{d} S} \cdot \hat{\boldsymbol{t}}\right)\left(\frac{\mathrm{d} \overline{\boldsymbol{u}}}{\mathrm{d} S} \cdot \hat{\boldsymbol{v}}\right)\right\}+ \\
& +\frac{1}{\|t\|^{2}}\left(\frac{\mathrm{d} \hat{\boldsymbol{t}}}{\mathrm{d} S} \cdot \hat{\boldsymbol{v}}\right)\left\{\left(\frac{\mathrm{d} \overline{\boldsymbol{u}}}{\mathrm{d} S} \cdot \hat{\boldsymbol{t}}\right)\left(\frac{\mathrm{d} \dot{\boldsymbol{u}}}{\mathrm{d} S} \cdot \hat{\boldsymbol{n}}\right)+\left(\frac{\mathrm{d} \dot{\boldsymbol{u}}}{\mathrm{d} S} \cdot \hat{\boldsymbol{t}}\right)\left(\frac{\mathrm{d} \overline{\boldsymbol{u}}}{\mathrm{d} S} \cdot \hat{\boldsymbol{n}}\right)\right\}+ \\
& +\frac{1}{\|\boldsymbol{t}\|^{2}}\left\{\left(\frac{\mathrm{d} \dot{\boldsymbol{u}}}{\mathrm{d} S} \cdot \hat{\boldsymbol{v}}\right)\left(\frac{\mathrm{d}^{2} \overline{\boldsymbol{u}}}{\mathrm{d} S^{2}} \cdot \hat{\boldsymbol{n}}\right)+\left(\frac{\mathrm{d} \dot{\boldsymbol{u}}}{\mathrm{d} S} \cdot \hat{\boldsymbol{v}}\right)\left(\frac{\mathrm{d} \overline{\boldsymbol{u}}}{\mathrm{d} S} \cdot \hat{\boldsymbol{t}}\right)\left(\frac{\mathrm{d} \hat{\boldsymbol{n}}}{\mathrm{d} S} \cdot \hat{\boldsymbol{t}}\right)\right\}+ \\
& -\frac{1}{\|\boldsymbol{t}\|^{2}}\left\{\left(\frac{\mathrm{d}^{2} \overline{\boldsymbol{u}}}{\mathrm{d} S^{2}} \cdot \hat{\boldsymbol{v}}\right)\left(\frac{\mathrm{d} \dot{\boldsymbol{u}}}{\mathrm{d} S} \cdot \hat{\boldsymbol{n}}\right)+\left(\frac{\mathrm{d} \dot{\boldsymbol{u}}}{\mathrm{d} S} \cdot \hat{\boldsymbol{n}}\right)\left(\frac{\mathrm{d} \overline{\boldsymbol{u}}}{\mathrm{d} S} \cdot \hat{\boldsymbol{t}}\right)\left(\frac{\mathrm{d} \hat{\boldsymbol{v}}}{\mathrm{d} S} \cdot \hat{\boldsymbol{t}}\right)\right\}
\end{aligned}
$$

Also, in this case, the first two terms of the Eq. (75) are symmetric in the their arguments, while the last two terms are not symmetric and are split in the symmetric and in the skew-symmetric parts, given by

$$
\partial_{\bar{u}} \partial_{\dot{\varphi}} \chi_{t}(\varphi)=\operatorname{sym}\left\{\partial_{\bar{u}} \partial_{\dot{\varphi}} \chi_{t}(\varphi)\right\}+\operatorname{skew}\left\{\partial_{\bar{u}} \partial_{\dot{\varphi}} \chi_{t}(\varphi)\right\}
$$

where the first is the sum of the first two terms in the right-hand side of the Eq. (75), while the skew-symmetric part, after some calculation, reduces to

$$
\text { skew }\left\{\partial_{\overline{\boldsymbol{u}}} \partial_{\dot{\varphi}} \chi_{t}(\varphi)\right\}=\hat{\boldsymbol{t}} \cdot \frac{d}{\mathrm{~d} S}\left(\nabla_{\overline{\boldsymbol{\omega}}} \dot{\boldsymbol{\omega}}\right) .
$$

\section{The tangent stiffness operator for the Kirchhoff rod model}

Collecting the results obtained, the geometric tangent operator defined by Eq. (58) can be expressed as

$$
\begin{aligned}
\mathbb{G}[\bar{\varphi}, \dot{\varphi}](\psi(\varphi))= & \int_{0}^{L} N(\boldsymbol{\varphi}) \partial_{\bar{\varphi}} \partial_{\dot{\varphi}} \varepsilon(\varphi) \mathrm{d} S+ \\
& +\int_{0}^{L} M_{n}(\varphi)\left(\operatorname{sym}\left\{\partial_{\bar{\varphi}} \partial_{\dot{\varphi}} \chi_{n}(\varphi)\right\}+\operatorname{skew}\left\{\partial_{\bar{\varphi}} \partial_{\dot{\varphi}} \chi_{n}(\varphi)\right\}\right) \mathrm{d} S+ \\
& +\int_{0}^{L} M_{\nu}(\varphi)\left(\operatorname{sym}\left\{\partial_{\bar{\varphi}} \partial_{\dot{\varphi}} \chi_{\nu}(\varphi)\right\}+\operatorname{skew}\left\{\partial_{\bar{\varphi}} \partial_{\dot{\varphi}} \chi_{v}(\varphi)\right\}\right) \mathrm{d} S+ \\
& +\int_{0}^{L} M_{t}(\varphi)\left(\operatorname{sym}\left\{\partial_{\bar{\varphi}} \partial_{\dot{\varphi}} \chi_{t}(\varphi)\right\}+\operatorname{skew}\left\{\partial_{\bar{\varphi}} \partial_{\dot{\varphi}} \chi_{t}(\varphi)\right\}\right) \mathrm{d} S,
\end{aligned}
$$

The explicit form of the skew-symmetric parts is

$$
\int_{0}^{L}\left(M_{t} \hat{\boldsymbol{t}} \cdot \frac{d}{\mathrm{~d} S}\left(\nabla_{\bar{\omega}} \dot{\boldsymbol{\omega}}\right)+\hat{\boldsymbol{t}} \times \boldsymbol{M} \cdot \boldsymbol{b}\left(\hat{\boldsymbol{t}} \cdot \nabla_{\bar{\omega}} \dot{\boldsymbol{\omega}}\right)\right) \mathrm{d} S .
$$


Integrating by part, one has

$$
\operatorname{skew}\{\mathbb{G}[\overline{\boldsymbol{\varphi}}, \dot{\boldsymbol{\varphi}}](\boldsymbol{\varphi})\}=\int_{0}^{L}\left[-\frac{d M_{t}}{\mathrm{~d} S}+\hat{\boldsymbol{t}} \times \boldsymbol{M} \cdot \boldsymbol{b}\right]\left(\hat{\boldsymbol{t}} \cdot \nabla_{\bar{\omega}} \dot{\boldsymbol{\omega}}\right) \mathrm{d} S .
$$

The equation in the bracket is the equilibrium equation dual to the torsional velocity of rotation given in Eq. (28).

In order to obtain the tangent stiffness, we still have to add the last term in Eq. (51) that represents the variation of the energy functional in the direction of the coefficients of structure. Since the only non-null connection symbol is given by the expression (47) that has only component along the $\hat{\boldsymbol{t}}$ direction, then the term $-\partial_{\partial_{\bar{\varphi}} \dot{\varphi}}(\psi)$ reduces to

$$
-\partial_{\nabla_{\bar{\varphi}} \dot{\varphi}}(\psi)=-\int_{0}^{L}\left[-\frac{\mathrm{d} M_{t}}{\mathrm{~d} S}+\hat{\boldsymbol{t}} \times \boldsymbol{M} \cdot \boldsymbol{b}\right]\left(\hat{\boldsymbol{t}} \cdot \nabla_{\overline{\boldsymbol{\omega}}} \dot{\boldsymbol{\omega}}\right) \mathrm{d} S .
$$

This term cancels out with (80), and then, the symmetry of the covariant Hessian is recovered.

\section{Conclusions and developments}

The consistent tangent stiffness operator for a Kirchhoff-Love rod model has been obtained performing the second covariant gradient of the elastic potential energy, assuming elastic behavior for the cross section. This has been done explicitly evaluating the Levi-Civita connection for the tangent space of the configurations, introducing a suitable metric that accounts for the rotations of the directors. The rotation operator for the Kirchhoff-Love rod has been split in two parts that induce a corresponding split in the rotation on the tangent space of the configurations. The only one coefficient of the defined connection different from zero in the case of Kirchhoff-Love rods is a torsional rotation, defined as the derivative of the bending rotation in the direction of the bending rotation.

The tangent operator evaluated in this way is symmetric for any pairs of admissible variations of the configuration of the rod. The result is essential for developing consistent numerical schemes for the nonlinear analysis of rods, where the consistent symmetric tangent operator is necessary in order to guarantee quadratic rate of convergence, and for buckling analysis.

The strong as well as the weak forms of the equilibrium equations have also been obtained, together with the boundary conditions that define the admissible boundary loads for the nonlinear Kirchhoff-Love beam model. The tangent kinematics has been stated, from which it is possible to obtain the discrete deformation operator in a numerical finite element model.

The results obtained in this paper will be the basis for developing an efficient numerical model for the nonlinear analysis of curved space rods, using the isogeometric interpolation proposed in [30,31]. The efficiency of the method, already examined for the linear case, will be tested when large deformations are attained, causing degradation of the properties of the tangent stiffness matrix. An interesting problem that will be investigated is the applicability of the method presented to the case of nonconservative forces and to the related buckling analysis of systems of rods with perturbation methods, as done in $[43,44,46,48]$, where the stability of a cantilever elastic beam under the action of a follower tangential force and a bending conservative couple at the free end was analyzed. Similar issues arise in the analysis of deformable curved beams under the action of wind loads.

\section{References}

1. Andreaus, U., Dell'Isola, F., Porfiri, M.: Piezoelectric passive distributed controllers for beam flexural vibrations. JVC/ J. Vib. Control 10(5), 625-659 (2004)

2. Antmann, S.S.: Nonlinear Problem of Elasticity. Springer, New York (1995)

3. Altenbach, H., Eremeyev, V.A.: On the effective stiffness of plates made of hyperelastic materials with initial stress. Int. J. Non-Linear Mech. 45(10), 976-981 (2010)

4. Alessandroni, S., Dell'Isola, F., Porfiri, M.: A revival of electric analogs for vibrating mechanical systems aimed to their efficient control by PZT actuators. Int. J. Solid Struct. 39(20), 5295-5324 (2002) 
5. Alibert, J.-J., Seppecher, P., Dell' Isola, F.: Truss modular beams with deformation energy depending on higher displacement gradients. Math. Mech. Solids 8(1), 51-73 (2003)

6. Argyris, J.: An excursion into large rotations. Comput. Methods Appl. Mech. Eng. 32, 85-155 (1982)

7. Atluri, S.N., Cazzani, A.: Rotations in computational solid mechanics. Arch. Comput. Methods Eng. 2, 49-138 (1995)

8. Bergou, M., Wardetzky, M., Robinson, S., Audoly, B., Grinspun, E.: Discrete elastic rod. ACM Trans. Graph. 27(3), 6316312 (2008)

9. Bersani, A.M., Giorgio, I., Tomassetti, G.: Buckling of an elastic hemispherical shell with an obstacle. Contin. Mech. Thermodyn. 25(2), 443-467 (2013)

10. Betsch, P., Steinmann, P.: Frame-indifferent beam finite elements based upon the geometrically exact beam theory. Int. J. Numer. Meth. Eng. 54, 1775-1788 (2002)

11. Birsan, M., Altenbach, H., Sadowski, T., Eremeyev, V.A., Pietras, D.: Deformation analysis of functionally graded beams by the direct approach. Compos. Part B Eng. 43(3), 1315-1328 (2012)

12. Crisfield, M.A., Jelenic, G.: Objectivity of strain measures in the geometrically exact three-dimensional beam theory and its finite-element implementation. Proc. R. Soc. Lond. 455, 1125-1147 (1999)

13. Crisfield, M.A.: Non-linear Finite Element Analysis of Solids and Structures, vol. 2, Advanced Topics. Wiley, New York (2000)

14. Cazzani, A.: On the dynamics of a beam partially supported by an elastic foundation: an exact solution-set. Int. J. Struct. Stab. Dyn. 13, 1350045 (2013). doi:10.1142/S0219455413500454

15. Cuomo, M., Greco, L. : Isogeometric analysis of space rods: Considerations on stress locking. In ECCOMAS, pp. 1-19 (2012)

16. Dell'Isola, F., Vidoli, S.: Continuum modeling of piezoelectromechanical truss beam: An application to vibration damping. Arch. Appl. Mech. 68(1), 1-19 (1998)

17. Dell'Isola, F., Porfiri, M., Vidoli, S.: Piezo-electromechanical (PEM) structures: Passive vibration control using distributed piezoelectric transducers. Comptes Rendus Mecanique 331(1), 69-76 (2003)

18. Dell'Isola, F., Seppecher, P.: The relationship between edge contact forces, double forces and interstitial working allowed by principle of virtual power. Comptes Rendus De Lacademie Des Sciences Serie II Fascicule B-Mecanique Physique Astronomie 321(8), 303-308 (1995)

19. Del Vescovo, D., Giorgio, I.: Dynamic problems for metamaterials: review of existing models and ideas for further research. Int. J. Eng. Sci, doi:10.1016/j.ijengsci.2014.02.002

20. Dell' Isola, F., Seppecher, P., Madeo, A.: How conctact interactions may depend on the shape of Cauchy cuts in Nth gradient continua: Approach “à la D’Alambert”. Zeitschrift für Angewandte Mathematik Und Physik 63(6), 1119-1141 (2012)

21. Eremeyev, V.A., Pietraszkiewicz, W.: Local symmetry group in the general theory of elastic shell. J. Elast. 85(2), 125152 (2006)

22. Eremeyev, V.A., Pietraszkiewicz, W.: Phase transitions in thermoelastic and thermoviscoelastic shells. Arch. Mech. 61(1), 41-67 (2009)

23. Eremeyev, V.A., Pietraszkiewicz, W.: The nonlinear theory of elastic shells with phase transitions. J. Elast. 74(1), 67$86(2004)$

24. Eremeyev, V.A., Pietraszkiewicz, W.: Thermomechanics of shells undergoing phase transition. J. Mech. Phys. 59(7), 1395$1412(2011)$

25. Eugster, S.R., Hesch, C., Betsch, P., Glocker, Ch.: Director-based beam finite elements relying on the geometrically exact beam theory formulated in skew coordinates. Int. J. Numer. Meth. Eng. doi:10.1002/nme.4586

26. Echter, R., Oesterle, B., Bischoff, M.: A hierarchic family of isogeometric shell finite elements. Comput. Methods Appl. Mech. Eng. 254, 170-180 (2013)

27. Ferretti, M., Madeo, A., Dell'Isola, F., Boisse, P.: Modeling the onset of shear boundary layers in fibrous composite reinforcements by second gradient theory. Zeitschrift für Angewandte Mathematik und Physik. doi:10.1007/s00033-013-03478

28. Fox, D.D., Simo, J.C.: A drill rotation formulation for geometrically exact shells. Comput. Methods Appl. Mech. Eng. 98, 329-343 (1992)

29. Giorgio, I., Culla, A., Del Vescovo, D.: Multimode vibration control using several piezoelectric transducers shunted with a multiterminal network. Arch. Appl. Mech. 79, 859-879 (2009)

30. Greco, L., Cuomo, M.: B-spline interpolation for Kirchhoff-Love space rod. Comput. Methods Appl. Mech. Eng. 256, 251269 (2012)

31. Greco, L., Cuomo, M.: An implicit $G^{1}$ multi patch B-spline interpolation for Kirchhoff love space rod. Comput. Methods Appl. Mech. Eng. 269, 173-197 (2014)

32. Ibrahimbegovic, A.: On the geometrically exact formulation of structural mechanics and its applications to dynamics, control and optimization. Comptes-Rendus Mecanique 331, 383-394 (2003)

33. Ibrahimbegovic, A.: Stress resultant geometrically nonlinear shell theory with drilling rotations-Part I. A consistent formulation. Comput. Methods Appl. Mech. Eng. 118, 265-284 (1994)

34. Ibrahimbegovic, A., Frey, F.: Stress resultant geometrically nonlinear shell theory with drilling rotations - Part II. Computational aspects. Comput. Methods Appl. Mech. Eng. 118, 285-308 (1994)

35. Jelenic, G., Saje, M.: A kinematically exact space finite strain beam model—finite element formulation by generalized virtual work principle. J. Appl. Math. Phys. 120, 131-161 (1995)

36. Jelenic, G., Crisfield, M.A.: Geometrically exact 3D beam theory: Implementation of a strain-invariant finite element for static and dynamics. Comput. Meth. Appl. Mech. Eng. 171, 141-171 (1999)

37. Langer, J., Singer, D.A.: Lagrangian aspect of the Kirchhoff elastic rod. SIAM Rev. 38(4), 605-618 (1996)

38. Luongo, A., Pignataro, M.: Multiple interaction and localization phenomenon in postbuckling of compressed thin-walled members. AIAA J. 26(11), 1395-1402 (1988)

39. Luongo, A., Pignataro, M.: On the perturbation analysis of interactive buckling in nearly symmetric structures. Int. J. Solids Struct. 29(6), 721-733 (1992) 
40. Luongo, A.: Perturbation methods for nonlinear autonomous discrete-time dynamic systems. Nonlinear Dyn. 10(4), $317-$ 331 (1996)

41. Luongo, A., Di Egidio, A., Paolone, A.: Multiple scale bifurcation analysis for finite-dimensional autonomous systems. Recent Res. Dev. Sound Vib. 1, 161-201 (2002)

42. Luongo, A.: Mode localization in dynamics and buckling of linear imperfect continuous structures. Nonlinear Dynamics 25(13), 133-156 (2001)

43. Luongo, A., D’Annibale, F.: Bifurcation analysis of damped visco-elastic planar beams under simultaneous gravitational and follower forces. Int. J. Modern Phys. B 26(25), 1246015-1-1246015-6 (2012)

44. Luongo, A., D’Annibale, F.: Double zero bifurcation of non-linear viscoelastic beams under conservative and nonconservative loads. Int. J. Non-Linear Mech. 55, 128-139 (2013)

45. Maurini, C., Pouget, J., Dell'Isola, F.: On a model of layered piezoelectric beams including transverse stress effect. Int. J. Solid Struct. 41(16-17), 4473-4502 (2004)

46. Paolone, A., Vasta, M., Luongo, A.: Flexural-torsional bifurcations of a cantilever beam under potential and circulatory forces: Part I Nonlinear model and stability analysis. Int. J. Non-Linear Mech. 41(4), 586-594 (2006)

47. Pietraszkiewicz, W., Eremeyev, V.A., Konopinska, V.: Extended non-linear relations of elastic shells undergoing phase transitions. Z. Angew. Math. Mech. (ZAMM) 87(2), 150-159 (2007)

48. Paolone, A., Vasta, M., Luongo, A.: Flexural-torsional bifurcations of a cantilever beam under potential and circulatory forces: Part II Post-critical analysis. Int. J. Non-Linear Mech. 41(4), 595-604 (2006)

49. Porfiri, M., Dell'Isola, F., Santini, E.: Modeling and design of passive electric networks interconnecting piezoelectric transducers for distributed vibration control. Int. J. Appl. Electromagn. Mech. 21(2), 69-87 (2005)

50. Porfiri, M., Dell'Isola, F., Mascioli, F.M.F.: Circuit analog of beam and its application to multimodal vibration damping, using piezoelectric transducers. Int. J. Circuit Theory Appl. 32(4), 167-198 (2004)

51. Reissner, E.: On finite deformations of space-curved beams. J. Appl. Math. Phys. 32, 734-744 (1981)

52. Rodrigues, O.: Des lois géométriques qui régissent les déplacements d'un systéme solide dans l'espace et de la variation des coornnées provenant de ces déplacements considéres indépendment des causes qui peuvent les produire. J. Math. Pures Appl. 5, 380-440 (1840)

53. Simo, J.C.: A finite beam formulation, The three dimensional dynamic I. Comput. Methods Appl. Mech. Eng. 49, 55$70(1985)$

54. Simo, J.C., Vu-Quoc, L.: A three-dimensional finite strain rod model, Part II: Computational aspects. Comput. Methods Appl. Mech. Eng. 58, 79-116 (1986)

55. Smolenski, WM: Statically and kinematically exact nonlinear theory of rods and its numerical verification. Comput. Methods Appl. Mech. Eng. 178, 89-113 (1999)

56. Simo, J.C., Vu-Quoc, L.: On the dynamics in space of rods undergoing large motions. A geometrically exact approach. Comput. Methods Appl. Mech. Eng. 66, 125-161 (1988)

57. Simo, J.C., Fox, D.D., Hughes, T.J.R.: Formulations of finite elasticity with independent rotations. Comput. Methods Appl. Mech. Eng. 95, 277-288 (1992)

58. Simo, J.C.: The (symmetric) Hessian for geometrically nonlinear models in solids mechanics: Intrinsic definition and geometric interpretation. Comput. Methods Appl. Mech. Eng. 96, 189-200 (1992)

59. Simo, J.C., Marsden, J.E., Krishnaprasad, P.S.: The Hamiltonian structure of nonlinear elasticity: The material and convective representations of solid, rods, and plates. Arch. Ration. Mech. Anal. 104(2), 125-183 (1988)

60. Simo, J.C., Fox, D.D.: On a stress resultant geometrically exact shell model. Part I: Formulation and optimal parametrization. Comput. Methods Appl. Mech. Eng. 72, 267-304 (1989)

61. Simo, J.C., Fox, D.D., Rifai, M.S.: On a stress resultant geometrically exact shell model. Part II: the linear theory; Computational aspects. Comput. Methods Appl. Mech. Eng. 58, 79-116 (1989)

62. Vidoli, S., Dell'Isola, F.: Modal coupling in one-dimensional electromechanical structured continua. Acta Mech. 141(1), 3750 (2000) Tuesday, April 29, 2014 at 4:34 pm 